Pacific

Journal of

Mathematics

REPRESENTATION TYPE OF COMMUTATIVE

NOETHERIAN RINGS I: LOCAL WILDNESS

Lee Klingler and Lawrence S. Levy 


\title{
REPRESENTATION TYPE OF COMMUTATIVE NOETHERIAN RINGS I: LOCAL WILDNESS
}

\author{
Lee Klingler and LaWrence S. Levy
}

\begin{abstract}
This is the first of a series of four papers describing the finitely generated modules over all commutative noetherian rings that do not have wild representation type (with a possible exception involving characteristic 2). This first paper identifies the wild rings, in the complete local case. The second paper describes the finitely generated modules over the remaining complete local rings. The last two papers extend these results by dropping the "complete local" hypothesis.
\end{abstract}

\section{Introduction.}

The goal of this project is to describe all finitely generated modules including the indecomposables and direct-sum behavior — over as wide as possible a class of commutative noetherian rings, thus extending Steinitz's well-known 1911 theorem on modules over Dedekind domains [S]. Steinitz's theorem has been particularly resistant to generalization. In fact, the only noetherian integral domains (other than Dedekind domains) for which such a module structure theorem was known prior to completion of this project seem to be the Dedekind-like domains studied in [L2]. However, for rings that are not domains, some other results exist $[\mathbf{L 1}, \mathbf{L 2}, \mathbf{N R}, \mathbf{N R S B}]$.

We call a ring $\Lambda$ finitely generated tame if we can describe all isomorphism classes of finitely generated $\Lambda$-modules. In all cases for which we cannot obtain such a description (with a possible exception involving characteristic 2 ), the obstruction is wild representation type; or more precisely, finitelength wildness.

Informally, a commutative ring $\Lambda$ is finite-length wild if it has a residue field $k$ such that any description of all isomorphism classes of $\Lambda$-modules of finite length would have to contain a description of all isomorphism classes of finite-dimensional modules over all finite-dimensional $k$-algebras. The seeming hopelessness of this task is what is behind the name "wild representation type." (The precise definition of finite-length wild is given in Subsection 2.2.) The notion of tame versus wild representation type has been important in the study of finite-dimensional algebras for more than 20 years but seems to be relatively new in commutative noetherian rings. So, 
we follow our formal Definition 2.2 by some introductory remarks on the subject [Remarks 2.3].

One of the surprising facts about finite dimensional algebras is that the majority of algebras over algebraically closed fields are wild. The well-known tame-wild theorem of Drozd and Crawley-Boevey [CB, Theorem B] states that, if $k$ is algebraically closed, then every finite dimensional $k$-algebra is either tame or wild, but never both.

One of the main objectives of the present series of papers is to obtain a similar tame-wild dichotomy for commutative noetherian rings. It is possible to give a precise definition of "tame" in the context of finite dimensional algebras over algebraically closed fields. (See, for example, [CB, Definition 6.5].) In the present series, however, "finitely generated tame" has the informal meaning stated in the second paragraph of this introduction.

For readability, this project is divided into a series of four papers.

Paper I: Wildness, complete local case. Let $(\Lambda, \mathfrak{m}, k)$ be a complete local commutative noetherian ring, and $\mu_{\Lambda}(\mathfrak{m})$ the minimal number of generators of the $\Lambda$-module $\mathfrak{m}$. We give the spirit of our Main Wildness Theorem [2.10] without the many definitions that are needed for its precise statement.

If $\mu_{\Lambda}(\mathfrak{m}) \geq 3$, then $\Lambda$ is always finite-length wild. On the other hand, if $\mu_{\Lambda}(\mathfrak{m})=1$, then $\Lambda$ is a principal ideal ring, and its tameness was well-known long before the word "tame" came into use. Thus $\mu_{\Lambda}(\mathfrak{m})=2$ is the dividing line between tameness and wildness; moreover, the vast majority of rings with $\mu_{\Lambda}(\mathfrak{m})=2$ are wild. In order to make this dividing line precise, we need to define several types of rings, which we do in Section 2.

We call $\Lambda$ an artinian triad if $\mu_{\Lambda}(\mathfrak{m})=3$ and $\mathfrak{m}^{2}=0$. These are clearly the "smallest" rings such that $\mu_{\Lambda}(\mathfrak{m})=3$, and they are known to be finite-length wild. We also define a special kind of local artinian ring $\Lambda$ of composition length 5 , with $\mu_{\Lambda}(\mathfrak{m})=2$, and call it a Drozd ring. We prove that these are finite-length wild in Section 4.

Section 3 is devoted to the proof of our Ring-theoretic Dichotomy Theorem [3.1], which states that every complete local ring either (i) maps onto an artinian triad or a Drozd ring, or (ii) is a homomorphic image of a type of ring of Krull dimension 1 that we call Dedekind-like or is an exceptional type of artinian ring that we call a Klein ring. "Dedekind-like rings" are reduced rings, satisfy $\mu_{\Lambda}(\mathfrak{m}) \leq 2$, and are very close to their normalization (in their total quotient ring), which is either a DVR (discrete valuation ring) or the direct sum of two DVRs. "Klein rings" have composition length 4 and satisfy $\mu_{\Lambda}(\mathfrak{m})=2$, a special case being the group algebra of the Klein 4-group over a field of characteristic 2. (See Section 2 for precise definitions.)

Our Ring-theoretic Dichotomy Theorem [3.1] is a piece of pure commutative algebra; neither it nor its proof make any use of the notions of tame 
or wild. In order to use this ring-theoretic dichotomy to prove our tamewild dichotomy, we need to prove that rings of type (ii) (in the previous paragraph) are tame. We postpone this to paper II.

Extending earlier terminology of Ringel $[\mathbf{R}]$, we think of artinian triads and Drozd rings as "minimal wild rings," and Dedekind-like rings and Klein rings as "maximal tame rings." Thus, anticipating the results of paper II, our dichotomy theorem implies that every $\Lambda$ either maps onto a minimal wild ring, and hence is finite-length wild, or is a homomorphic image of a maximal tame ring, and hence is finitely-generated tame. (The possible exception involving characteristic 2 is described in our discussion of paper II below.)

Paper I ends with three short sections containing examples and miscellaneous results:

Section 5 gives an example of a Klein ring that is not an algebra over a field, and studies when Klein rings are, and when they are not, homomorphic images of Dedekind-like rings.

Section 6 gives a second, more constructive definition of Drozd rings than the abstract definition in Subsection 2.4. This section also shows that all of the ramified complete local orders studied in integral representation theory are finite-length wild.

Section 7 shows that all complete local orders of infinite lattice type are finite-length wild.

Remarks on earlier work. Let $\Lambda$ be a commutative noetherian ring that is a finitely generated algebra over an algebraically closed field. In this context, our tame-wild dichotomy for $\Lambda$-modules of finite length was obtained by Drozd $[\mathbf{D}]$, and the solution improved by Ringel $[\mathbf{R}]$. The actual module structure in the tame case for modules of finite length - in fact, for finitely generated modules - had been given by earlier results of others ( $[\mathbf{N R}]$, corrected in $[\mathbf{N R S B}])$. This collection of results was the source of our interest in the subject.

As Drozd and Ringel were only interested in $\Lambda$-modules of finite length, they were able to assume, without loss of generality, that $\Lambda$ is a complete local ring. Since our rings do not have to be algebras over fields and do not have to be local rings, our results on finitely generated modules apply to rings of algebraic integers, the rings that originally interested Steinitz. Our results also appear to be new in the situation that $\Lambda$ is an artinian ring that is not an algebra over a field, and in the case of algebras over nonalgebraically-closed fields. (On the other hand, Ringel's paper does contain noncommutative results which we do not handle.)

As mentioned above, the original Nazarova-Roiter results in $[\mathbf{N R}]$ deal with finitely generated modules over the local rings that they investigated, a 
special case of our Dedekind-like rings. But since Drozd's paper, the "finitelength wild versus finitely-generated tame" aspect of the problem seems to have been mostly neglected.

In a later paper [D2], Drozd states a tame-wild dichotomy over a class of rings that can be noncommutative and need not be algebras over a field. However, most of the statements and all of the proofs of theorems in that paper assume that the ring is an algebra over an algebraically closed field. Thus, in the commutative case, the results proved in that paper do not go beyond those in his earlier paper. Moreover, in the commutative case, his unproved statement of which rings are tame does not take the simple, explicit form that is given by our definition of split and unsplit Dedekind-like rings and Klein rings. (See our $\S 2$.)

Paper II: Tameness, complete local case [KL2]. This long paper gives a detailed description of all finitely generated modules over Klein rings and over complete local Dedekind-like rings, with the possible exception described in (1.0.1). Except for this possible exception, this completes our finitelygenerated tame versus finite-length wild dichotomy for complete local rings $\Lambda$.

(1.0.1) The possible exception. There are three types of Dedekind-like rings: split, unsplit, and DVRs (see Definition 2.5 below). Let $\Lambda$ be a complete, unsplit Dedekind-like ring, with maximal ideal $\mathfrak{m}$ and residue field $k$, and let $\Gamma$ be the normalization of $\Lambda$. By definition of "unsplit Dedekindlike," $\mathfrak{m}$ is also the unique maximal ideal of $\Gamma$, and $\Gamma / \mathfrak{m}$ is a quadratic field extension of $k=\Lambda / \mathfrak{m}$. When the quadratic field extension $\Gamma / \mathfrak{m}$ of $k$ is inseparable, our theory breaks down, and we do not know whether $\Lambda$ is tame or wild or neither.

Note, however, that this exception cannot occur for rings of algebraic integers (whose residue fields are finite) nor for rings of geometric origin (whose residue fields are algebraically closed).

Paper III: Global Wildness [KL3]. In this short paper we remove the "complete local" hypothesis from the main wildness theorem of Paper I. We may assume, without loss of generality, that we are given a commutative, noetherian, indecomposable ring $\Omega$. Our main result is that, if $\Omega$ is not finite-length wild, then $\Omega$ is either a homomorphic image of a "global Dedekind-like ring" $\Lambda$ (that is, a reduced ring of Krull dimension 1, all of whose completions $\hat{\Lambda}_{\mathfrak{m}}$ at maximal ideals are the local Dedekind-like rings, as previously defined), or $\Omega$ is a Klein ring.

As in paper I, we postpone the statements and proofs of the corresponding tameness results to the next paper in the series.

Paper IV: Global Tameness [KL4]. Let $\Lambda$ be a global Dedekind-like ring, as defined in the discussion of paper III above. We describe the structure of 
all finitely-generated $\Lambda$-modules, provided that no completion $\hat{\Lambda}_{\mathfrak{m}}$ is one of the possible exceptions involving characteristic 2 , described in (1.0.1).

Here it is not sufficient to describe the indecomposable $\Lambda$-modules. Since mod- $\Lambda$, the category of all finitely generated $\Lambda$-modules, is not a KrullSchmidt category, we also need to describe the direct-sum relations of finitely generated $\Lambda$-modules. Indeed, this and the local-global relations in mod- $\Lambda$ occupy most of this rather long paper.

One additional and possibly surprising complication is that - unlike rings of number-theoretic or geometric origin - it is not necessarily true that all but finitely many completions $\hat{\Lambda}_{\mathfrak{m}}$ of our non-local Dedekind-like ring $\Lambda$ be DVRs. In fact, it is possible for $\Lambda$ to have infinitely many maximal ideals and none of its completions be DVRs.

An interesting application of our structure theory is that every ring of the form $\mathbb{Z}[\sqrt{n}]$, with $n$ a square-free integer, is among the tame rings whose finitely generated modules we describe. (Note that $\mathbb{Z}[\sqrt{n}]$ is not always the full ring of algebraic integers in $\mathbb{Q}[\sqrt{n}]$.)

\section{Definitions, main theorem.}

In this section we define finite-length wildness and the types of rings that appear in the statement of our Main Wildness Theorem [2.10], and give some examples. Then we state the theorem itself and give some additional examples.

Notation 2.1. Throughout this paper, $\Lambda$ denotes a commutative noetherian ring. We say that $(\Lambda, \mathfrak{m}, k)$ is a local ring if $\Lambda$ is a noetherian ring with unique maximal ideal $\mathfrak{m}$ and residue field $k$. We say that $(\Lambda, \mathfrak{m}, k)$ is complete if it is $\mathfrak{m}$-adically complete.

We consistently write functions on the left except when they represent matrix multiplication. It is typographically simpler to display a row of a matrix than a column. Therefore our matrices normally act via right multiplication, and we write the corresponding functions as right operators. This occurs throughout this series of papers, and we include a reminder when it happens.

We let $\mu_{\Lambda}(M)$ denote the minimal number of generators required by a $\Lambda$-module $M$.

Definition 2.2 (Finite-length wildness). Let fdmod- $k\langle X, Y\rangle$ denote the category of finite dimensional right modules over the free noncommutative $k$-algebra in two indeterminates $X$ and $Y$.

We say that $\Lambda$ is finite-length wild (with respect to $k$ ) if $k$ is a residue field of $\Lambda$, and there is a full subcategory $\mathcal{W}$ of the category of $\Lambda$-modules of finite length and an additive functor $\Phi: \mathcal{W} \rightarrow$ fdmod- $k\langle X, Y\rangle$ such that $\Phi$ is a representation equivalence; that is, $\Phi$ is: dense (onto all isomorphism classes), faithful $(\Phi(M) \cong \Phi(N) \Longleftrightarrow M \cong N)$, and full (a surjection 
on homomorphism groups). Thus, encoded in $\mathcal{W}$ we find not only all of the isomorphism classes in fdmod- $k\langle X, Y\rangle$, but (after reducing the homomorphism groups in $\mathcal{W}$ modulo suitable kernels) all of the hom-structure of fdmod- $k\langle X, Y\rangle$ as well.

\section{Remarks 2.3 (Introduction to wildness).}

(i) (Meaning of wildness) Every module over any 2-generator $k$-algebra $A$ is also a $k\langle X, Y\rangle$-module. Therefore fdmod- $k\langle X, Y\rangle$ contains fdmod- $A$ as a subcategory. Moreover, a well-known simple but clever trick of Brenner [B, Theorem 3] shows that fdmod- $k\langle X, Y\rangle$ contains a copy of fdmod- $A$, for every finitely generated $k$-algebra $A$. Thus, any classification of all isomorphism classes in fdmod- $k\langle X, Y\rangle$ would contain a classification of all isomorphism classes of finite dimensional $A$-modules for every finitely generated $k$-algebra A.

Suppose, now, that our commutative ring $\Lambda$ is finite-length wild, and let $M$ and $N$ be finite-dimensional modules over some 2-generator algebra $A$. We wish to know whether $M \cong N$. By density of the functor $\Phi$ in our definition of finite-length wild, we have $M \cong \Phi\left(M^{\prime}\right)$ and $N \cong \Phi\left(N^{\prime}\right)$ for $\Lambda$ modules $M^{\prime}, N^{\prime} \in \mathcal{W}$. Moreover, by faithfulness of $\Phi$ we have that $M^{\prime} \cong N^{\prime}$ as $\Lambda$-modules if and only if $M \cong N$ as $k\langle X, Y\rangle$-modules; or equivalently, as $A$-modules. Moreover, in this illustration of the meaning of wildness, we can drop the requirement that $A$ be 2-generated, by using Brenner's trick.

Thus we see that, if $\Lambda$ is finite length wild, then any explicit description of all isomorphism classes of $\Lambda$-modules of finite length would have to contain a description of all isomorphism classes of finite-dimensional A-modules, for every finite dimensional $k$-algebra $A$, as mentioned in the informal definition of wildness given in the introduction to this paper.

(ii) (Wildness of $\Lambda$ versus its localizations and completions) Let $\mathfrak{m}$ be a maximal ideal of $\Lambda$, and suppose that the $\mathfrak{m}$-localization $\Lambda_{\mathfrak{m}}$ or $\mathfrak{m}$-adic completion $\hat{\Lambda}_{\mathfrak{m}}$ is finite-length wild. Then $\Lambda$ is also finite-length wild. The reason for this is that every $\hat{\Lambda}_{\mathfrak{m}}$-module of finite length and every $\Lambda_{\mathfrak{m}}$-module of finite length is also a $\Lambda$-module of finite length.

Therefore, in order to prove that $\Lambda$ is finite-length wild, we can assume that $(\Lambda, \mathfrak{m}, k)$ is a complete local ring, the situation considered in this paper.

(iii) (Strict wildness) A $k$-algebra $A$ is sometimes called strictly wild if there is a full exact imbedding $\Psi$ : fdmod- $k\langle X, Y\rangle \rightarrow$ fdmod- $A$. This means that $\Psi$ is an isomorphism on hom groups, takes exact sequences in fdmod- $k\langle X, Y\rangle$ to exact sequences in fdmod- $A$, and is an imbedding of categories.

Definition 2.4 (Artinian triad, Drozd ring). We call the local ring $(\Lambda, \mathfrak{m}$, $k$ ) an artinian triad if $\mu_{\Lambda}(\mathfrak{m})=3$ and $\mathfrak{m}^{2}=0$. (Since $\mathfrak{m}^{2}=0$, every artinian triad is indeed an artinian ring.) In the case of finite dimensional algebras 
over the field $k=\Lambda / \mathfrak{m}$, this ring is $\Lambda=k[X, Y, Z] /(X, Y, Z)^{2}$, where $X, Y$, and $Z$ are indeterminates.

We call the local ring $(\Lambda, \mathfrak{m}, k)$ a Drozd ring if $\mu_{\Lambda}(\mathfrak{m})=\mu_{\Lambda}\left(\mathfrak{m}^{2}\right)=2$, $\mathfrak{m}^{3}=0$, and there is an element $x \in \mathfrak{m}-\mathfrak{m}^{2}$ such that $x^{2}=0$. (Since $\mathfrak{m}^{3}=0$, Drozd rings are artinian.) In the case of finite dimensional algebras over the field $k=\Lambda / \mathfrak{m}$, this ring is the 5 -dimensional $k$-algebra with $k$-basis $1, x, y, x y, y^{2}$ and all other monomials equal to zero. An example of a Drozd ring that is not a algebra over a field is the ring $A_{p}$ in Example 6.1.

We note:

(2.4.1) The composition length of every Drozd ring is 5 .

To see this note that the dimensions of $\Lambda / \mathfrak{m}, \mathfrak{m} / \mathfrak{m}^{2}$, and $\mathfrak{m}^{2}$ as $k$-vector spaces are $1,2,2$ respectively.

The above, abstract definition of Drozd rings is tailored to the needs of our dichotomy results, but leaves one wondering what these rings really look like. It turns out that every Drozd ring is a subring of an artinian principal ideal ring, and we use this fact to give a general, explicit construction of Drozd rings in Theorem 6.5.

Definition 2.5 (Dedekind-like ring). Let $(\Lambda, \mathfrak{m}, k)$ be a local ring. We call $\Lambda$ a Dedekind-like ring if $\Lambda$ is reduced (no nonzero nilpotent elements) and its normalization $\Gamma$ (in the total quotient ring of $\Lambda$ ) has the following properties: $\Gamma$ is a direct sum of principal ideal domains (necessarily semi-local), $\mathfrak{m}=$ $\operatorname{rad}(\Gamma)$ (the Jacobson radical of $\Gamma$ ), and $\mu_{\Lambda}(\Gamma) \leq 2$.

We do not consider fields to be principal ideal domains. Therefore: $\Gamma$ and $\Lambda$ have Krull dimension 1.

This abstract definition is tailored to the needs of the proof of our dichotomy theorem. For a more constructive definition and examples, see Notation 2.13 and the lemmas and examples that follow it.

Note that the reduced ring $\Gamma / \mathfrak{m}$ is a vector space over $k=\Lambda / \mathfrak{m}$ of dimension at most 2 . Therefore exactly one of the following three possibilities holds, and we attach the indicated name to $\Lambda$.

(2.5.1) We call the Dedekind-like ring $\Lambda$ :

(i) Split if $\Gamma / \mathfrak{m} \cong k \times k$ as rings.

(ii) Unsplit if $\Gamma / \mathfrak{m}$ is a 2-dimensional field extension of $k$. (We usually call this field $F$.)

(iii) A DVR (discrete valuation ring) if $\Gamma / \mathfrak{m}=k$; that is, $\Lambda=\Gamma$.

Since $\Gamma / \mathfrak{m}$ has $k$-dimension at most 2 and $\mathfrak{m}=\operatorname{rad}(\Gamma), \Gamma$ must be either an integral domain or the direct sum of two integral domains. We attach names to the corresponding possibilities as follows.

(2.5.2) We call the split Dedekind-like ring $\Lambda$ :

(i) Strictly split if $\Gamma$ is the direct sum of two integral domains (necessarily DVRs). 
(ii) Nonstrictly split if $\Gamma$ is an integral domain (necessarily a PID with exactly two maximal ideals).

We note: If the split Dedekind-like ring $\Lambda$ is complete, then $\Lambda$ is strictly split. To see this, note first that ${ }_{\Lambda} \Gamma$ is $\mathfrak{m}$-adically complete, since ${ }_{\Lambda} \Gamma$ is finitely generated ([N, Theorem 17.8]). Since the ideal $\mathfrak{m}$ of $\Lambda$ also equals $\operatorname{rad}(\Gamma)$, we see that the semilocal ring $\Gamma$ is also $\mathfrak{m}$-adically complete, and therefore a direct sum of local rings. The fact that $\Lambda$ is strictly split now follows from (2.5.2).

If $\Lambda$ is unsplit then $\Gamma$ is a DVR, because $\Gamma / \mathfrak{m}=\Gamma / \operatorname{rad}(\Gamma)$ is a field.

Remark 2.6 (Consistency with terminology of [L2]). All localizations and completions at maximal ideals of the rings called "Dedekind-like" in [L2] are split Dedekind-like, in our present terminology [by Lemma 2.14 below], or DVRs. Thus, for local rings, our present terminology generalizes that of [L2]. When we consider the non-local situation in [KL3] and [KL4], our terminology will again generalize that in $[\mathbf{L 2}]$.

In order to prove that Dedekind-like rings that are not DVRs lie on the tame-wild dividing line mentioned in the introduction, we show:

Lemma 2.7. If $(\Lambda, \mathfrak{m}, k)$ is Dedekind-like but not a DVR, then $\mu_{\Lambda}(\mathfrak{m})=2$.

Proof. By Nakayama's Lemma it suffices to show that the $k$-vector space $\mathfrak{m} / \mathfrak{m}^{2}$ has dimension 2. Since $\mathfrak{m}$ is also an ideal of the principal ideal ring $\Gamma$, we have $\mathfrak{m} / \mathfrak{m}^{2} \cong \Gamma / \mathfrak{m}$ as $\Gamma$-modules, and hence as $\Lambda$-modules, and therefore as $k$-vector spaces. This last dimension equals 2 except if $\Lambda$ is a DVR, by (2.5.1).

Definition 2.8 (Klein ring). We call a local ring $(\Lambda, \mathfrak{m}, k)$ a Klein ring if $\mu_{\Lambda}(\mathfrak{m})=2, \mu_{\Lambda}\left(\mathfrak{m}^{2}\right)=1, \mathfrak{m}^{3}=0$, and $x^{2}=0(\forall x \in \mathfrak{m})$. (Since $\mathfrak{m}^{3}=0$, Klein rings are artinian and hence definitely not reduced.)

The group algebra of the Klein 4-group over a field of characteristic 2 is an example of a Klein ring. We also note:

Lemma 2.9. If $\Lambda$ is a Klein ring with residue field $k$, then $k$ has characteristic 2 , and $\Lambda$ has characteristic 2 or 4 .

Proof. Let $\mathfrak{m}=(x, y)$. Then $0=(x+y)^{2}=x^{2}+2 x y+y^{2}=2 x y$. Since $\mu_{\Lambda}\left(\mathfrak{m}^{2}\right)=1$ and $x^{2}=y^{2}=0$, we cannot have $x y=0$. Therefore $2 x y=0$ implies that $2 \in \mathfrak{m}$. Since $\Lambda$ is a Klein ring, this implies that $4=2^{2}=0$ in $\Lambda$, completing the proof.

For an example in which characteristic 4 actually occurs, see Example 5.4.

Having now defined all of the ingredients needed to state our main theorem, we now state the theorem itself. 
Theorem 2.10 (Main Wildness Theorem). Let $(\Lambda, \mathfrak{m}, k)$ be a complete local ring. Then exactly one of the following holds.

(i) $\Lambda$ maps onto an artinian triad or a Drozd ring, in which case $\Lambda$ is finite-length wild.

(ii) $\Lambda$ is either a Klein ring or a homomorphic image of a strictly split or unsplit Dedekind-like ring.

Proof. Our Ring-theoretic Dichotomy Theorem 3.1 states that either (ii) holds, or else $\Lambda$ maps onto an artinian triad or onto a Drozd ring. Since finitelength wildness clearly carries up from homomorphic images, it therefore suffices to prove that artinian triads and Drozd rings are finite-length wild.

Artinian triads are wild by a theorem of Warfield [GLW, Lemma 3]; and Drozd rings are wild by Theorem 4.9.

Remarks 2.11. (i) As previously mentioned, we finish the complete local case of our tame-wild project in the second paper of this series [KL2] by describing the structure of all finitely generated $\Lambda$-modules when $\Lambda$ is Dedekind-like or a Klein ring and the possible exception (1.0.1) does not occur.

(ii) There exist Klein rings that are not homomorphic images of Dedekindlike rings (Theorem 5.2). Therefore, we cannot simplify Theorem 2.10 by deleting the phrase "Klein ring" from statement (ii). On the other hand, some Klein rings are homomorphic images of Dedekind-like rings (again, Theorem 5.2).

(iii) The reason that DVRs can be omitted from the statement of Theorem 2.10(ii) is that every DVR is a homomorphic image of a strictly split Dedekind-like ring [Lemma 2.19].

(iv) Note that Theorem 2.10 does not state that $\Lambda$ cannot be both tame and wild. For finite-dimensional algebras over an algebraically closed field, the terms "tame" and "wild" are known to be mutually exclusive [CB, Theorem B]. In the context of commutative noetherian rings, the question remains open.

Corollary 2.12. Every noetherian ring $\Lambda$ (not necessarily local) of Krull dimension greater than one is finite-length wild.

Proof. Since $\Lambda$ has dimension greater than $1, \Lambda$ has a maximal ideal $\mathfrak{m}$ such that the $\mathfrak{m}$-localization $\Lambda_{\mathfrak{m}}$ has dimension greater than 1 , and hence $[\mathbf{N}$, 17.12] the $\mathfrak{m}$-adic completion $\hat{\Lambda}_{\mathfrak{m}}$ has dimension greater than 1 . Therefore we may assume that $\Lambda$ itself is a complete local ring of dimension greater than 1 [Remarks 2.3(ii)].

Dedekind-like rings have Krull dimension 1, and Klein rings are artinian, hence have Krull dimension 0 . Therefore, by Theorem $2.10, \Lambda$ is finite-length wild. 
It is often more informative to view Dedekind-like rings in terms of their conductor square. The last few results in this section deal with this point of view.

Notation 2.13 (Conductor Square). Consider the following commutative diagram of ring homomorphisms, where $\mathfrak{m}$ is an ideal of both rings $\Lambda$ and $\Gamma$ (a "conductor" ideal).

$$
\begin{aligned}
& \Lambda \subseteq \Gamma \\
& \|^{\Lambda} \rho \quad{ }^{\infty} \quad(\operatorname{ker} \rho=\mathfrak{m}) . \\
& k \subseteq \bar{\Gamma}=\Gamma / \mathfrak{m}
\end{aligned}
$$

We say that $\Lambda$ is the pullback of this conductor square if $\Lambda=\{x \in \Gamma \mid \rho(x) \in$ $k\}$.

The next three simple lemmas make clear exactly what ingredients are needed to construct arbitrary Dedekind-like rings.

Lemma 2.14 (Split Dedekind-like). Let $\Gamma$ be a principal ideal ring with exactly two maximal ideals and no artinian ring direct summands. Let $\mathfrak{m}=\operatorname{rad}(\Gamma)$ (the Jacobson radical of $\Gamma$ ), and suppose that $\Gamma / \mathfrak{m} \cong k \times k$, the direct product of two copies of some field $k$. View $k$ as a subfield of $k \times k$ via the diagonal map $x \rightarrow(x, x)$, and let $\Lambda$ be the pullback of square (2.13.1). Then $(\Lambda, \mathfrak{m}, k)$ is a split Dedekind-like ring with normalization $\Gamma$, and every split Dedekind-like ring is isomorphic to such a $\Lambda$.

Proof. The only part of this proof that requires explicit mention is perhaps to note that we have $\mu_{\Lambda}(\Gamma) \leq 2$ because the $k$-dimension of $\bar{\Gamma}$ is 2 ; and therefore the integrally closed reduced ring $\Gamma$ is the normalization of $\Lambda$.

The next lemma is particularly useful in when the local ring $\Lambda$ is complete, since in that situation all split Dedekind-like rings are strictly split.

Lemma 2.15 (Strictly Split Dedekind-like). Let $\left(V_{1}, \mathfrak{m}_{1}, k\right)$ and $\left(V_{2}, \mathfrak{m}_{2}, k\right)$ be DVRs with a common residue field $k$. For each $i$ let $f_{i}: V_{i} \rightarrow k$ be a surjective ring homomorphism, and let

$$
\Lambda=\left\{\left[v_{1}, v_{2}\right] \in V_{1} \times V_{2} \mid f_{1}\left(v_{1}\right)=f_{2}\left(v_{2}\right)\right\} .
$$

Then $\left(\Lambda,\left(\mathfrak{m}_{1} \times \mathfrak{m}_{2}\right), k\right)$ is a strictly split Dedekind-like ring with normalization $\Gamma=V_{1} \times V_{2}$. Moreover, every strictly split Dedekind-like ring is isomorphic to such a ring $\Lambda$.

Proof. To verify the first assertion, build conductor square (2.13.1) as follows. Let $\bar{\Gamma}=k \times k$ and view $k$ as a subring of $\bar{\Gamma}$ via the diagonal map $x \rightarrow(x, x)$. Let $\mathfrak{m}=\mathfrak{m}_{1} \times \mathfrak{m}_{2}$ and $\rho=f_{1} \times f_{2}$. Then we can identify $\rho(\Gamma)$ with $\Gamma / \mathfrak{m}$. Now use Lemma 2.14

The proof of the next lemma is essentially the same as that of Lemma 2.14. 
Lemma 2.16 (Unsplit Dedekind-like). Let $\Gamma$ be a DVR. Let $\mathfrak{m}=\operatorname{rad}(\Gamma)$, suppose that $\Gamma / \mathfrak{m}$ is a 2-dimensional field extension of some subfield $k$, and let $\Lambda$ be the pullback of square (2.13.1). Then $(\Lambda, \mathfrak{m}, k)$ is an unsplit Dedekind-like ring with normalization $\Gamma$, and every unsplit Dedekind-like ring is isomorphic to such a $\Lambda$.

Although the class of Dedekind-like rings is very small, it contains many interesting examples; we give a few below. For more examples, and tameness properties of Dedekind-like rings, see [KL2].

\section{Examples 2.17 (Split).}

(i) The simplest example of a strictly split Dedekind-like ring is formed by taking $V_{1}$ and $V_{2}$ to be the $p$-localization or $p$-adic completion of the integers, for some prime number $p$, in Lemma 2.15. Note that this is not an algebra over a field.

For the minor modification that is an algebra over any field $k$, use Lemma 2.15 and take $V_{1}$ and $V_{2}$ to be $k[[X]]$ (formal power series).

(ii) The ring $k[[X, Y]] /(X \cdot Y)$ is strictly split Dedekind-like. To see this, note that every element can be represented by an ordered pair of power series in one variable, where both power series have the same constant term. Thus this is isomorphic to the example constructed in the second paragraph of item (i) above.

(iii) Let $\Lambda=\hat{\mathbb{Z}}_{p}[[X]] /(p X)$ where $p$ is any prime number and $\hat{\mathbb{Z}}_{p}$ denotes the $p$-adic integers. Then $\Lambda$ is a strictly split Dedekind-like ring. One can view this as the number-theoretic analog of the ring $k[[X, Y]] /(X Y)$ in item (ii).

To prove this, let $V_{1}=\hat{\mathbb{Z}}_{p}[[X]] /(X) \cong \hat{\mathbb{Z}}_{p}$ and $V_{2}=\hat{\mathbb{Z}}_{p}[[X]] /(p) \cong \overline{\mathbb{Z}}_{p}[[X]]$, both of which are complete DVRs. The natural map $\nu: \Lambda \rightarrow V_{1} \oplus V_{2}$ is clearly one-to-one, and it is not difficult to verify that $\nu$ maps $\Lambda$ onto the split Dedekind-like ring described in (2.15.1) and Lemma 2.15, with $k=$ $\overline{\mathbb{Z}}_{p}=\mathbb{Z} /(p)$ and $f_{1}$ and $f_{2}$ the natural homomorphisms.

(iv) (Nonstrictly split). Let $\Gamma$ be any principal ideal domain that has exactly two maximal ideals $\mathfrak{m}_{1}$ and $\mathfrak{m}_{2}$ with isomorphic residue fields $k$. (For example start with the ring of algebraic integers in any quadratic extension of the rationals, and localize away all but two maximal ideals that lie over some common rational prime.) Then let $\Lambda$ be the pullback of diagram (2.13.1) in which $\rho$ is a surjective ring homomorphism $\Gamma \rightarrow k \times k$ with kernel $\mathfrak{m}=\mathfrak{m}_{1} \cap \mathfrak{m}_{2} . \Lambda$ is split Dedekind-like by Lemma 2.14, and is not strictly split since it is an integral domain.

We remind the reader that nonstrictly split Dedekind-like rings can never be complete local rings.

Examples 2.18. The easiest example of an unsplit Dedekind-like ring is the ring $\Lambda=\mathbb{R}+X \mathbb{C}[[X]]$ of power series whose constant terms are real 
numbers and whose other coefficients are complex numbers. To see this, use Lemma 2.16 with $\Gamma=\mathbb{C}[[X]], \bar{\Gamma}=\mathbb{C}$, and $k=\mathbb{R}$.

For an example that is not an algebra over a field, let $\Gamma^{\prime}$ be the ring of all integers in any number field such that some residue field $F$ of $\Gamma^{\prime}$ is a 2dimensional extension of some subfield $k$. Then let $\Gamma$ be the localization or completion of $\Gamma^{\prime}$ with respect to the kernel of $\Gamma^{\prime} \rightarrow F$. Finish the definition by letting $\Lambda$ be the pullback of diagram (2.13.1) and using Lemma 2.16.

Finally, for future reference, we record three useful lemmas.

Lemma 2.19. Let $\Omega$ be either a DVR or an artinian local principal ideal ring. Then $\Omega$ is a homomorphic image of a strictly split Dedekind-like ring $\Lambda$. Moreover, if $\Omega$ is a complete DVR or is artinian, then $\Lambda$ can be chosen to be complete.

Proof. Let $(V, \mathfrak{n}, k)$ be a DVR, and $f: V \rightarrow k$ the natural homomorphism. Then let

$$
\Lambda=\{[x, y] \in V \times V \mid f(x)=f(y)\} .
$$

The ring $\Lambda$ is strictly split Dedekind-like, by Lemma 2.15 , and maps onto $V$. Moreover, if $V$ is complete, then $\Lambda$ is also complete.

Let $A$ be an artinian local principal ideal ring. A theorem of Hungerford $[\mathbf{H u}]$ states that some complete DVR $V$ maps onto $A$. Since $\Lambda$ maps onto $V$, the proof is complete.

Lemma 2.20. Let $(\Lambda, \mathfrak{m}, k)$ be a strictly split Dedekind-like ring. Then $\mathfrak{m}$ is generated by two elements $x, y$ such that $x y=0$.

Proof. View $\Lambda$ in the notation of Lemma 2.15. Then take generators $p_{1}$ and $p_{2}$ of the maximal ideals of $V_{1}$ and $V_{2}$ respectively. Finally, note that the elements $x=\left[p_{1}, 0\right]$ and $x=\left[0, p_{2}\right]$ of $V_{1} \times V_{2}$ are elements of $\Lambda$ and, in fact, generate $\mathfrak{m}$.

Lemma 2.21. Let $(\Lambda, \mathfrak{m}, k)$ be an unsplit or split Dedekind-like ring with normalization $\Gamma$. Then the $\mathfrak{m}$-adic completion $(\hat{\Lambda}, \hat{\mathfrak{m}}, k)$ is, respectively, an unsplit or (necessarily strictly) split Dedekind-like ring with normalization $\hat{\Gamma}$, and $\operatorname{rad} \hat{\Gamma}=\hat{\mathfrak{m}}$.

Proof. Since $\mathfrak{m}$ is an ideal of both rings $\Lambda$ and $\Gamma$, the $\mathfrak{m}$-adic completion of $\Gamma$ as a $\Lambda$-module coincides with the $\mathfrak{m}$-adic completion of $\Gamma$ as a $\Gamma$-module. Therefore we can denote either by $\hat{\Gamma}$. Moreover, since $\Gamma$ is a finitely generated $\Lambda$-module, its $\mathfrak{m}$-adic completion as a $\Lambda$-module can be identified with $\hat{\Lambda} \otimes_{\Lambda} \Gamma$; that is, $\hat{\Gamma}=\hat{\Lambda} \otimes_{\Lambda} \Gamma$.

Viewing $\hat{\Gamma}$ as the $\mathfrak{m}$-adic completion as a $\Gamma$-module, we see that $\hat{\Gamma}$ is a DVR or the direct sum of two DVRs according as $\Lambda$ is, respectively, unsplit or split. 
Now tensor pullback diagram (2.13.1) for $\Lambda$ by $\hat{\Lambda}$ over $\Lambda$, remembering that tensoring a pullback diagram by a flat module yields a pullback diagram (use the idea in e.g., [K, Proposition 2.10] or [L2, Lemma 6.1]). We claim that the resulting pullback diagram shows that $\Lambda$ is the appropriate kind of Dedekind-like ring. In view of what has already been proved, we only need to note two more facts: (i) The finite-length modules in the bottom row of the original pullback diagram are unchanged by completion; and (ii) $\operatorname{ker}(\hat{\rho})=\operatorname{rad}(\hat{\Gamma})$. To prove this last fact, call the kernel $K$. For every $x \in K$, the element $1-x$ is invertible since $\hat{\mathfrak{m}}$ is the radical of the local $\operatorname{ring} \operatorname{rad} \hat{\Lambda}$, and therefore $K \subseteq \operatorname{rad}(\hat{\Gamma})$. The opposite inclusion holds since $\hat{\rho}(\hat{\Gamma})$ has radical zero.

\section{Dichotomy theorem.}

The purpose of this section is to prove the following result.

Theorem 3.1 (Ring-theoretic Dichotomy). Let $(\Lambda, \mathfrak{m}, k)$ be a complete, local ring. Then exactly one of the following two possibilities holds.

(i) $\Lambda$ has an artinian triad or a Drozd ring as homomorphic image.

(ii) $\Lambda$ is a Klein ring or a homomorphic image of a complete, strictly split or unsplit Dedekind-like ring.

The remainder of this section is devoted to the proof of this theorem, which we accomplish by means of a series of propositions.

Remark 3.2. We begin by noting that, if $\mu_{\Lambda}(\mathfrak{m}) \geq 3$, then clearly $\Lambda$ maps onto an artinian triad. On the other hand, if $\mu_{\Lambda}(\mathfrak{m}) \leq 1$, then by a theorem of Hungerford $[\mathbf{H u}], \Lambda$ is a homomorphic image of a complete DVR $(\Gamma, \mathfrak{m}, k)$, and hence a homomorphic image of a complete, strictly split Dedekind-like ring (Lemma 2.19). Therefore, for the remainder of this section we can assume that $\mu_{\Lambda}(\mathfrak{m})=2$.

Thus, we can write $\mathfrak{m}=(x, y)$, so that $\mathfrak{m}^{2}=\left(x^{2}, x y, y^{2}\right)$, and hence $\mu_{\Lambda}\left(\mathfrak{m}^{2}\right) \leq 3$. If in fact $\mu_{\Lambda}\left(\mathfrak{m}^{2}\right)=3$, then $\Lambda /\left(\left(x^{2}\right)+\mathfrak{m}^{3}\right)$ is a Drozd ring. Therefore, for the remainder of this section, we can also assume that $\mu_{\Lambda}\left(\mathfrak{m}^{2}\right)$ $\leq 2$.

The argument continues as we attempt to use generators $x$ and $y$ of $\mathfrak{m}$ and the relations satisfied by $x^{2}, x y$, and $y^{2}$ to construct a strictly split or unsplit Dedekind-like ring that maps onto $\Lambda$.

Lemma 3.3. Let $(\Lambda, \mathfrak{m}, k)$ be a complete, local ring, and suppose that $\mu_{\Lambda}(\mathfrak{m})$ $=2$ and $\mu_{\Lambda}\left(\mathfrak{m}^{2}\right) \leq 2$. If $x$ and $y$ are generators of the maximal ideal $\mathfrak{m}$, and if $x y \in \mathfrak{m}^{3}$, then we can find generators $u$ and $v$ of $\mathfrak{m}$ such that $u v=0$.

Proof. Note that for each integer $n \geq 2$ we have $\mathfrak{m}^{n}=\left(x^{n}, x^{n-1} y, \ldots, x y^{n-1}\right.$, $\left.y^{n}\right)$. But $x y \in \mathfrak{m}^{3}$, so it follows that $x^{n-1} y, \ldots, x y^{n-1} \in \mathfrak{m}^{n+1}$, and hence by Nakayama's Lemma we have $\mathfrak{m}^{n}=\left(x^{n}, y^{n}\right)$. 
The strategy is to construct sequences $u_{1}, u_{2}, u_{3}, \ldots$ and $v_{1}, v_{2}, v_{3}, \ldots$ of elements of $\Lambda$, such that

$$
\mathfrak{m}=\left(u_{n}, v_{n}\right), \quad u_{n} v_{n} \in \mathfrak{m}^{n+2}, \quad \text { and } \quad u_{n}-u_{n+1}, \quad v_{n}-v_{n+1} \in \mathfrak{m}^{n+1}
$$

for each index $n$. It then follows immediately that the sequences are Cauchy sequences and, if we let $u=\lim _{n \rightarrow \infty}\left(u_{n}\right)$ and $v=\lim _{n \rightarrow \infty}\left(v_{n}\right)$, that $\mathfrak{m}=$ $(u, v)$ and $u v=0$.

The construction of the sequences is by induction on $n$. For $n=1$, set $u_{1}=x$ and $v_{1}=y$. Now suppose, inductively, that we have selected elements $u_{1}, \ldots, u_{n}$ and $v_{1}, \ldots, v_{n}$ of $\Lambda$ satisfying (3.3.1).

As noted above, we have $\mathfrak{m}^{n+2}=\left(u_{n}^{n+2}, v_{n}^{n+2}\right)$. Thus we can write

$$
u_{n} v_{n}=a u_{n}^{n+2}+b v_{n}^{n+2}
$$

for some $a, b \in \Lambda$. Rearranging (3.3.2) yields

$$
u_{n}\left(v_{n}-a u_{n}^{n+1}\right)=b v_{n}^{n+2} .
$$

Therefore setting $v_{n+1}=v_{n}-a u_{n}^{n+1}$ gives a new pair of generators $\mathfrak{m}=$ $\left(u_{n}, v_{n+1}\right)$ such that $v_{n}-v_{n+1} \in \mathfrak{m}^{n+1}$. Now (3.3.3) can be rewritten as

$$
u_{n} v_{n+1}=b\left(v_{n+1}+a u_{n}^{n+1}\right)^{n+2} .
$$

Expanding the right-hand side and rearranging yields

$$
\left(u_{n}-b v_{n+1}^{n+1}\right) v_{n+1} \in \mathfrak{m}^{n+3} .
$$

Therefore setting $u_{n+1}=u_{n}-b v_{n+1}^{n+1}$ completes the induction, and hence the proof of the lemma.

Under the conditions of this technical lemma, we are ready to construct a complete, strictly split Dedekind-like ring that maps onto $\Lambda$.

Proposition 3.4. Let $(\Lambda, \mathfrak{m}, k)$ be a complete local ring, such that $\mu_{\Lambda}(\mathfrak{m})=$ 2 , and suppose that $x$ and $y$ are a pair of generators of $\mathfrak{m}$ such that $x y \in \mathfrak{m}^{3}$. Then $\Lambda$ is a homomorphic image of a complete, strictly split Dedekind-like ring.

Proof. By Lemma 3.3, we can assume that $x y=0$.

By the Structure Theorem of Complete Local Rings [N, Theorem 31.1 and preceding paragraph], there is a surjective homomorphism $\phi: V[[X, Y]] \rightarrow \Lambda$ such that $\phi(X)=x$ and $\phi(Y)=y$, where $V[[X, Y]]$ is a formal power series ring, and either $V=k$ or $V$ is a complete DVR of characteristic 0 with residue field $k$. Moreover, in this latter case, $k$ has characteristic $p \neq 0$, and the maximal ideal of $V$ is generated by $p$.

If $V$ is a field, let us set $R=V[[X, Y]]$, a complete, two-dimensional, regular local ring.

If $V$ is not a field, we note that $V[[X, Y]]$ is a complete, local, threedimensional domain with maximal ideal $(p, X, Y)$, so that $V[[X, Y]]$ is a 
regular local ring (by $[\mathbf{A M}$, Theorem 11.22]) and therefore a unique factorization domain. Now $\phi(p) \in \mathfrak{m}$ since $k$ has characteristic $p$, and hence there are elements $A, B \in V[[X, Y]]$ such that $\phi(p-(A X+B Y))=0$. Since $p$ is irreducible in $V$, clearly $p-(A X+B Y)$ is irreducible in $V[[X, Y]]$, and hence $(p-(A X+B Y))$ is a prime ideal. We set $R=V[[X, Y]] /(p-(A X+B Y))$, a complete, local, two-dimensional domain by [AM, Corollary 11.18]. Moreover, the maximal ideal of $R$ is now generated by two elements (the images of $X$ and $Y$ ), so that $R$ is in fact a regular local ring in this case also (again by [AM, Theorem 11.22]). Changing notation, we let $\phi$ denote the induced map from $R$ onto $\Lambda$, and we denote by $X, Y$ the cosets of the original elements $X, Y$, respectively, in $R$.

Thus, in either case, we have a surjective homomorphism $\phi: R \rightarrow \Lambda$ such that $\phi(X)=x$ and $\phi(Y)=y$, where $R$ is a complete, two-dimensional, regular local ring, $(X, Y)$ is the maximal ideal of $R$, and $R /(X, Y) \cong k$.

Now $\phi(X Y)=x y=0$, so that $\phi$ induces a surjective homomorphism from $R /(X Y)$ onto $\Lambda$. But the regular local ring $R$ is a unique factorization domain, and $X$ and $Y$ are non-associate primes (being generators of the twogenerated maximal ideal of $R)$. Therefore, $(X Y)=(X) \cap(Y)$ as ideals of $R$. We set $V_{1}=R /(X)$ and $V_{2}=R /(Y)$. Each of $V_{1}$ and $V_{2}$ is a complete, local domain of Krull dimension one (since $X$ and $Y$ are irreducible), and each has a principal maximal ideal (since we mod out by the other generator), so that both $V_{1}$ and $V_{2}$ are complete DVRs.

Finally, by [CR1, 2.12], there is a pullback diagram with surjective maps:

$$
\begin{array}{ccc}
R /(X Y)=R /((X) \cap(Y)) & V_{2}=R /(Y) \\
V_{1}=R /(X) & f_{2} \downarrow
\end{array}
$$

That is, $R /(X Y)$ is isomorphic to the pullback

$$
\Omega=\left\{[s, t] \in V_{1} \times V_{2} \mid f_{1}(s)=f_{2}(t)\right\}
$$

of the pair of (natural) maps $f_{1}$ and $f_{2}$. Since $V_{1}$ and $V_{2}$ are DVRs and $k$ is a field, the ring $R /(X Y) \cong \Omega$ is strictly split Dedekind-like by Lemma 2.15 . Since $\phi: R \rightarrow \Lambda$ is a surjective ring homomorphism whose kernel contains $(X Y)$, our proof is complete.

Given the result of Proposition 3.4, we can assume that, for every pair of generators $x$ and $y$ of the maximal ideal $\mathfrak{m}$ of $\Lambda, x y \notin \mathfrak{m}^{3}$. We also recall that we assume that $\mu_{\Lambda}\left(\mathfrak{m}^{2}\right) \leq 2$. The next step is to reduce to the case where $\mu_{\Lambda}\left(\mathfrak{m}^{2}\right)=2$.

Proposition 3.5. Let $(\Lambda, \mathfrak{m}, k)$ be a complete, local ring, such that $\mu_{\Lambda}(\mathfrak{m})=$ 2 , and suppose that $x y \notin \mathfrak{m}^{3}$ for every pair of generators $x$ and $y$ of $\mathfrak{m}$. Then either $\mu_{\Lambda}\left(\mathfrak{m}^{2}\right) \geq 2$, or $\Lambda$ is a Klein ring. 
Proof. Since we assume that $x y \notin \mathfrak{m}^{3}$ for every pair of generators $x$ and $y$ of $\mathfrak{m}$, clearly $\mu_{\Lambda}\left(\mathfrak{m}^{2}\right) \geq 1$. Thus, we can assume that $\mu_{\Lambda}\left(\mathfrak{m}^{2}\right)=1$, and it suffices to show that $\Lambda$ is a Klein ring.

Fix a pair of generators $x$ and $y$ of $\mathfrak{m}$. By assumption, $\mu_{\Lambda}\left(\mathfrak{m}^{2}\right)=1$ and $x y \notin \mathfrak{m}^{3}$, so that in fact $\mathfrak{m}^{2}=(x y)$. Thus, $x^{2} \in(x y)$, say $x^{2}=a x y$ for some element $a \in \Lambda$. But then $x(x-a y)=0$, and if $a$ were a unit in $\Lambda$, then we could replace $y$ by $x$-ay to get a pair of generators of $\mathfrak{m}$ whose product is 0 , contrary to assumption. Therefore, $a \in \mathfrak{m}$, and hence $x^{2} \in \mathfrak{m}^{3}$. Similarly, $y^{2} \in \mathfrak{m}^{3}$. But now $\mathfrak{m}^{3}=\left(x^{3}, x^{2} y, x y^{2}, y^{3}\right) \subseteq \mathfrak{m}^{4}$ implies, by Nakayama's Lemma, that $\mathfrak{m}^{3}=0$. In particular, $x^{2}=y^{2}=0$.

To complete the proof that $\Lambda$ is a Klein ring, we must show that $z^{2}=0$ for every element $z \in \mathfrak{m}$. If we write $z=a x+b y$ for some elements $a, b \in \Lambda$, with $x$ and $y$ the fixed generators of $\mathfrak{m}$ from above, then $z^{2}=a^{2} x^{2}+2 a b x y+b^{2} y^{2}=$ $2 a b x y$, so that it suffices to show that the residue field of $\Lambda$ has characteristic 2. But if the residue field of $\Lambda$ were not of characteristic 2 , then $x+y$ and $x-y$ would be generators of $\mathfrak{m}$, where $(x+y)(x-y)=x^{2}-y^{2}=0$, contrary to assumption.

Given the result of Proposition 3.5, we can assume that $\mu_{\Lambda}(\mathfrak{m})=\mu_{\Lambda}\left(\mathfrak{m}^{2}\right)$ $=2$ and that $x y \notin \mathfrak{m}^{3}$, for every pair of generators $x$ and $y$ of $\mathfrak{m}$. If, for some element $x \in \mathfrak{m}-\mathfrak{m}^{2}$, we had $x^{2} \in \mathfrak{m}^{3}$, then by definition $\Lambda / \mathfrak{m}^{3}$ would be a Drozd ring. Therefore, we can also assume that $x^{2} \notin \mathfrak{m}^{3}$ for every element $x \in \mathfrak{m}-\mathfrak{m}^{2}$. We are now ready to map an unsplit Dedekind-like ring onto $\Lambda$.

Proposition 3.6. Let $(\Lambda, \mathfrak{m}, k)$ be a complete local ring such that $\mu_{\Lambda}(\mathfrak{m})=$ $\mu_{\Lambda}\left(\mathfrak{m}^{2}\right)=2$. Suppose that $x y \notin \mathfrak{m}^{3}$ for every pair of generators $x$ and $y$ of $\mathfrak{m}$, and $x^{2} \notin \mathfrak{m}^{3}$ for every element $x \in \mathfrak{m}-\mathfrak{m}^{2}$. Then $\Lambda$ is a homomorphic image of a complete unsplit Dedekind-like ring.

Proof. Fix generators $x$ and $y$ of $\mathfrak{m}$. By assumption, $\mu_{\Lambda}\left(\mathfrak{m}^{2}\right)=2$, and $x y \notin \mathfrak{m}^{3}$. Thus, we can choose $x y$ as one of the two generators of $\mathfrak{m}^{2}$. Without loss of generality we can assume that $\mathfrak{m}^{2}=\left(x^{2}, x y\right)$. Hence we can write

$$
y^{2}+s x y+t x^{2}=0
$$

for some elements $s, t \in \Lambda$. Let $\bar{s}$ and $\bar{t}$ denote the images of $s$ and $t$, respectively, in $k=\Lambda / \mathfrak{m}$.

We claim that the polynomial $Z^{2}+\bar{s} Z+\bar{t}$ is irreducible in the polynomial ring $k[Z]$. Suppose it were reducible, say $Z^{2}+\bar{s} Z+\bar{t}=(Z+\bar{u})(Z+\bar{v})$, where $\bar{u}$ and $\bar{v}$ are the images in $k$ of the elements $u, v \in \Lambda$, respectively. This would imply that $y^{2}+s x y+t x^{2}-(y+u x)(y+v x) \in \mathfrak{m}^{3}$. Then by (3.6.1) we would have $(y+u x)(y+v x) \in \mathfrak{m}^{3}$. On the one hand, if $u-v \in \Lambda-\mathfrak{m}$, then $y+u x$ and $y+v x$ would be generators of $\mathfrak{m}$ (because their difference would be a unit times $x$ ), but their product is in $\mathfrak{m}^{3}$, contrary to the hypotheses 
of the proposition. On the other hand, if $u-v \in \mathfrak{m}$, then it would follow that $(y+u x)^{2} \in \mathfrak{m}^{3}$. But $x$ and $y$ are linearly independent modulo $\mathfrak{m}^{2}$, since they generate $\mathfrak{m}$ and $\mu_{\Lambda}(\mathfrak{m})=2$. Therefore $y+u x \in \mathfrak{m}-\mathfrak{m}^{2}$, and this again contradicts the hypotheses of the proposition. Therefore the claim holds.

We continue as in the proof of Proposition 3.4. Using the Structure Theorem of Complete Local Rings [N, Theorem 31.1], there is a surjective homomorphism $\phi: V[[X, Y]] \rightarrow \Lambda$ such that $\phi(X)=x$ and $\phi(Y)=y$, where $V[[X, Y]]$ is a formal power series ring, and either $V=k$ or $V$ is a complete DVR of characteristic 0 with residue field $k$, and where, in this latter case, $k$ has characteristic $p \neq 0$ and the maximal ideal of $V$ is generated by $p$.

If $V$ is a field, we set $R=V[[X, Y]]$. If $V$ is not a field, we set $R=$ $V[[X, Y]] /(p-(A X+B Y))$, where $A, B \in V[[X, Y]]$ are elements such that $\phi(p-(A X+B Y))=0$. In the latter case we change notation: Denote again by $\phi$ the induced map from $R$ onto $\Lambda$, and by $X$ and $Y$ the cosets in $R$ of $X$ and $Y$, respectively. Then, exactly as in the proof of Proposition 3.4, $\phi: R \rightarrow \Lambda$ is a surjective homomorphism such that $\phi(X)=x$ and $\phi(Y)=y$, where $R$ is a complete two-dimensional regular local ring and $\mathfrak{m}_{R}=(X, Y)$ is the maximal ideal of $R$, and of course $\phi$ induces an isomorphism between the residue fields of $R$ and $\Lambda$. Note that (3.6.1) still holds since $\Lambda$ has not changed.

Now select elements $S, T \in R$ such that $\phi(S)=s$ and $\phi(T)=t$. We claim that $Y^{2}+S X Y+T X^{2}$ is an irreducible element in the unique factorization domain $R$. Suppose, by way of contradiction, that $Y^{2}+S X Y+T X^{2}=C D$ in $R$, where neither $C$ nor $D$ is a unit. We know that $\mu_{R}\left(\mathfrak{m}_{R}^{2}\right)=3$ (by $[\mathbf{A M}$, Theorem 11.22]), and $\mathfrak{m}_{R}^{2}=\left(X^{2}, X Y, Y^{2}\right)$, so that $C D=Y^{2}+S X Y+T X^{2} \in$ $\mathfrak{m}_{R}^{2}-\mathfrak{m}_{R}^{3}$. Note that $\phi(C) \phi(D)=\phi\left(Y^{2}+S X Y+T X^{2}\right)=0$ by (3.6.1). But $\phi\left(\mathfrak{m}_{R}\right)=\mathfrak{m}$, and both maximal ideals require two generators, from which we get a contradiction as follows. If $C$ and $D$ are linearly independent modulo $\mathfrak{m}_{R}^{2}$, then they generate $\mathfrak{m}_{R}$, so that $\phi(C)$ and $\phi(D)$ are generators of $\mathfrak{m}$ such that $\phi(C) \phi(D)=0$, contrary to the hypotheses of the proposition. If $C$ and $D$ are linearly dependent modulo $\mathfrak{m}_{R}^{2}$, then they are associates, from which it follows that $\phi(C)^{2}=0$, again contrary to the hypotheses of the proposition. Therefore the claim holds.

We set $\Omega=R /\left(Y^{2}+S X Y+T X^{2}\right)$. Since $\phi\left(Y^{2}+S X Y+T X^{2}\right)=0$, the map $\phi$ factors through $\Omega$ to yield a surjective homomorphism from $\Omega$ onto $\Lambda$; moreover, this homomorphism induces an isomorphism of the residue fields of $\Omega$ and $\Lambda$. To complete the proof of the proposition, we need only show that $\Omega$ is an unsplit Dedekind-like ring.

First we recall that $R$ is a unique factorization domain, and $Y^{2}+S X Y+$ $T X^{2}$ is irreducible, so that $\left(Y^{2}+S X Y+T X^{2}\right)$ is prime, and therefore $\Omega$ is a (complete local noetherian one-dimensional) domain. Since we need only concern ourselves with the ring $\Omega$ for the remainder of this proposition, let us denote by $x, y, s$, and $t$ the images in $\Omega$ of the elements $X, Y, S$, and 
$T$, respectively, of $R$. Let us also denote by $\mathfrak{m}$ the maximal ideal of $\Omega$, and note that $\mathfrak{m}=(x, y)$. By the definition of $\Omega$, we have $y^{2}+s x y+t x^{2}=0$; that is, (3.6.1) still holds. Therefore $(y / x)^{2}+s(y / x)+t=0$ in the quotient field of $\Omega$, and hence $y / x$ is integral over $\Omega$ in its quotient field. Let us set $\Gamma=\Omega[y / x]=\Omega+\Omega \cdot y / x$ (using the fact that $(y / x)^{2}=-s(y / x)-t$ ).

Now $\Gamma$ is a finite, integral extension of $\Omega$ in its quotient field, so that $\Gamma$ is a semilocal integral domain. We claim that $\mathfrak{m}$ is an ideal of $\Gamma$. It suffices to prove that $\mathfrak{m}$ is closed under multiplication by $y / x$. We have $(y / x) x=y \in \mathfrak{m}$ and, by (3.6.1), $(y / x) y=-\left(s x y+t x^{2}\right) / x=-s y-t x \in \mathfrak{m}$, and thus the claim is proved.

Let $F=\Gamma / \mathfrak{m}$. We claim that $F$ is a 2-dimensional field extension of $k$. Since $\mathfrak{m}$ is an ideal of $\Gamma, F=\Gamma / \mathfrak{m}$ is a ring containing $k=\Lambda / \mathfrak{m}$. Now, $F$ is generated as a $k$-algebra by the image $\alpha$ of $y / x$ in $F$. As proved below (3.6.1), the polynomial $Z^{2}+\bar{s} Z+\bar{t}$ is irreducible over $k$. Since the $\alpha$ is a zero of this polynomial, by (3.6.1), we see that $F$ is a 2-dimensional field extension of $k$, as claimed.

Next we claim that $\mathfrak{m}=\operatorname{rad}(\Gamma)$. For the inclusion $(\subseteq)$ it suffices to prove that, for every element $m$ of the ideal $\mathfrak{m}$ of $\Gamma, 1-m$ is invertible in $\Gamma$; and this follows from the fact that $\mathfrak{m}=\operatorname{rad}(\Omega)$. The inclusion $(\supseteq)$ holds since $F=\Gamma / \mathfrak{m}$ is a field.

$\Gamma$ is a local ring since $\Gamma / \operatorname{rad}(\Gamma)$ is a field. We have $\mathfrak{m}=\Gamma(\Lambda x+\Lambda y)=\Gamma x$ because $y=(y / x) x \in \Gamma x$. Since the maximal ideal $\mathfrak{m}$ of the local domain $\Gamma$ is principal, we see that $\Gamma$ is a DVR. Thus $\Gamma$ is integrally closed and therefore must be the normalization of $\Omega$. Finally, we note that $\mu_{\Omega}(\Gamma)=2$, so that, by Definition 2.5, $\Omega$ is unsplit Dedekind-like.

We remark that, since $\Gamma$ is finitely generated as an $\Omega$-module, $\Gamma$ is complete in the $\mathfrak{m}$-adic topology as an $\Omega$-module [N, Theorem 17.8]. But since $\mathfrak{m}$ is also an ideal of $\Gamma$, this is the same as the $\mathfrak{m}$-adic topology on $\Gamma$ as a ring. Therefore, $(\Gamma, \mathfrak{m}, F)$ is a complete DVR.

Remark 3.2 and Propositions 3.4, 3.5, and 3.6 together show that, for a given complete, local ring $(\Lambda, \mathfrak{m}, k)$, at least one of the conditions (i) or (ii) of Theorem 3.1 must hold. It remains to show that these two conditions are mutually exclusive, which we show in the final lemma and proposition of this section.

Lemma 3.7. Let $(\Lambda, \mathfrak{m}, k)$ be a complete Dedekind-like ring, but not a DVR.

(i) If $w \in \mathfrak{m}-\mathfrak{m}^{2}$ then $w^{2} \notin \mathfrak{m}^{3}$.

(ii) The (composition) length of the ring $\Lambda / \mathfrak{m}^{3}$ is 5 .

Proof. Let $\Gamma$ be the normalization of $\Lambda$.

(i) The important idea here is that $\mathfrak{m}=\operatorname{rad}(\Gamma)$ and therefore both the hypothesis $w \in \mathfrak{m}-\mathfrak{m}^{2}$ and the conclusion $w^{2} \notin \mathfrak{m}^{3}$ say the same thing 
whether we consider $\mathfrak{m}$ to be an ideal of $\Lambda$ or of $\Gamma$. So we work in the ring $\Gamma$ instead of $\Lambda$.

Case 1: $\Lambda$ unsplit Dedekind-like. Then $\Gamma$ is a DVR and hence the desired conclusion is obvious.

Case 2: $\Lambda$ strictly split Dedekind-like. Here $\Gamma=V_{1} \times V_{2}$, the direct product of two DVRs and $\mathfrak{m}=\operatorname{rad}(\Gamma)=\operatorname{rad}\left(V_{1}\right) \times \operatorname{rad}\left(V_{2}\right)$; and so the desired conclusion follows easily from the fact that each $V_{i}$ is a DVR.

(ii) The bottom row of conductor square (2.13.1) shows that $\Gamma / \Lambda$ is a simple $\Lambda$-module. Therefore so is $\left(\Gamma / \mathfrak{m}^{3}\right) /\left(\Lambda / \mathfrak{m}^{3}\right)$. So it suffices to show that $\Gamma / \mathfrak{m}^{3}$ has length 6 as a $\Lambda$-module.

Since $\Gamma$ is either a DVR or the direct product of two DVRs, and $\mathfrak{m}=$ $\operatorname{rad}(\Gamma)$, it suffices to show that $\Gamma / \mathfrak{m}$ has length 2 as a $\Lambda$-module; that is, as a $k$-vector space. See (2.5.1).

To show that conditions (i) and (ii) of Theorem 3.1 are mutually exclusive, clearly it suffices to show that Klein rings and complete, strictly split or unsplit Dedekind-like rings cannot be mapped onto either an artinian triad or a Drozd ring. This we show in the final proposition of this section, which completes the proof of the theorem.

Proposition 3.8. Let $(\Lambda, \mathfrak{m}, k)$ be either a Klein ring or a complete, strictly split or unsplit Dedekind-like ring. Then $\Lambda$ does not have either an artinian triad or a Drozd ring as homomorphic image.

Proof. Since $\mu_{\Lambda}(\mathfrak{m})=2$, clearly $\Lambda$ cannot map onto an artinian triad. Similarly, if $\Lambda$ is a Klein ring, then $\mu_{\Lambda}\left(\mathfrak{m}^{2}\right)=1$, and hence $\Lambda$ cannot map onto a Drozd ring.

Therefore, we can suppose that $\Lambda$ is a complete split or unsplit Dedekindlike ring, and we must show that $\Lambda$ cannot map onto a Drozd ring. Suppose, by way of contradiction, that $\phi: \Lambda \longrightarrow A$ were a homomorphism from $\Lambda$ onto the Drozd ring $A$. Then $\phi\left(\mathfrak{m}^{3}\right)=\phi(\mathfrak{m})^{3}=0$ implies that $\phi$ would induce a map from $\Lambda / \mathfrak{m}^{3}$ onto $A$. Since both $\Lambda / \mathfrak{m}^{3}$ and $A$ have composition length five (by Lemma 3.7 and (2.4.1)), we would obtain the isomorphism $\Lambda / \mathfrak{m}^{3} \cong A$. That is, $\Lambda / \mathfrak{m}^{3}$ would be a Drozd ring.

But then $\Lambda$ would contain an element $w$ whose image $\bar{w}$ in $\Lambda / \mathfrak{m}^{3}$ satisfies $\bar{w} \in \overline{\mathfrak{m}}-\overline{\mathfrak{m}}^{2}$ and $\bar{w}^{2}=0$. Therefore $w \in \mathfrak{m}-\mathfrak{m}^{2}$ and $w^{2} \in \mathfrak{m}^{3}$, contrary to Lemma 3.7.

\section{Wildness of Drozd rings.}

The purpose of this section is to prove its final result, Theorem 4.9, establishing finite-length wildness of Drozd rings.

Caution. When functions represent matrix multiplication, we write the functions as right-hand operators. This occurs many times in this section. 
Notation 4.1. Recall (see Definition 2.4) that a Drozd ring is an artinian local ring $(\Lambda, \mathfrak{m}, k)$ such that $\mu_{\Lambda}(\mathfrak{m})=\mu_{\Lambda}\left(\mathfrak{m}^{2}\right)=2, \mathfrak{m}^{3}=0$, and $(\exists x \in$ $\left.\mathfrak{m}-\mathfrak{m}^{2}\right) x^{2}=0$. Since $\mu_{\Lambda}(\mathfrak{m})=2$ we have $\mathfrak{m}=(x, y)$ for some $y \in \mathfrak{m}-\mathfrak{m}^{2}$. Fix such elements $x, y$ for the rest of this section. Then $\mathfrak{m}^{2}=\left(x y, y^{2}\right)$, and the only nonzero monomials in $x$ and $y$ are $x, y, x y, y^{2}$.

In Drozd's original paper [D], $\Lambda$ is a 5 -dimensional $k$-algebra, and $1, x, y$, $x y, x^{2}$ form a basis of $\Lambda$. In our more general setting we partially repair the lack of a basis by establishing the following standard form for elements of $\mathfrak{m}$.

Lemma 4.2. For all elements $c \in \mathfrak{m}$, we can express $c=u_{1} x+u_{2} y+u_{3} x y+$ $u_{4} y^{2}$ with each $u_{i}$ a unit or 0 . (Note that we do not claim uniqueness of the coefficients $u_{i}$.)

Proof. There is an expression $c=a x+b y$. We can write $a=u_{1}+a^{\prime} x+b^{\prime} y$ where $u_{1}$ is a unit or 0 . (If $u_{1}$ is a unit, we can take $a^{\prime}=0=b^{\prime}$, but this is not important.) Similarly, $b=u_{2}+a^{\prime \prime} x+b^{\prime \prime} y$. Then $c=u_{1} x+u_{2} y+\left(b^{\prime}+\right.$ $\left.a^{\prime \prime}\right) x y+b^{\prime \prime} y^{2}$. Making similar substitutions for $b^{\prime}+a^{\prime \prime}$ and $b^{\prime \prime}$ completes the proof.

Viewing $\Lambda /(y)$ and $\Lambda /(x y)$ as $\Lambda$-modules, for $\lambda \in \Lambda$ we denote by $\lambda_{y}$ (respectively $\lambda_{x y}$ ) the coset of $\lambda$ in $\Lambda /(y)$ (respectively $\Lambda /(x y)$ ). Thus, $\Lambda /(y)=\Lambda 1_{y}$ has submodule $\Lambda x_{y}$, etc. The following lemma establishes some basic facts about $\Lambda$ and its homomorphic images $\Lambda 1_{y}$ and $\Lambda 1_{x y}$.

Lemma 4.3. (i) All of the monomials $x, x y, x_{x y}, \ldots$ in diagram (4.3.1) below are nonzero.

(ii) $\operatorname{soc} \Lambda 1_{y}=\Lambda x_{y}$, $\operatorname{soc} \Lambda 1_{x y}=\Lambda x_{x y} \oplus \Lambda y_{x y}^{2}$, and $\operatorname{soc} \Lambda=\Lambda x y \oplus \Lambda y^{2}=\mathfrak{m}^{2}$.

(iii) $\Lambda x \cap \Lambda y=\Lambda x y$ and $\Lambda x_{x y} \cap \Lambda y_{x y}=0$.

(iv) $\Lambda x \cong \Lambda y_{x y}$ as $\Lambda$-modules via the multiplication map $c x \rightarrow c y_{x y}$.

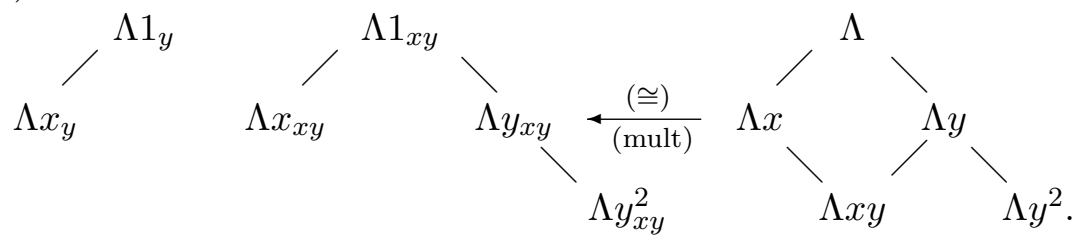

Proof. $\Lambda$. Since $\mathfrak{m}^{2}=\left(x y, y^{2}\right)$ and $\mu_{\Lambda}\left(\mathfrak{m}^{2}\right)=2$, the monomials displayed in $\Lambda$ are nonzero. To prove the nontrivial inclusion in $\Lambda x \cap \Lambda y=\Lambda x y$, assume that $c x=d y$. The element $c$ cannot be a unit, since this would imply $\mu_{\Lambda}(x, y)=1$. Therefore we can write $c$ in the standard form of Lemma 4.2, getting $c x=u_{2} x y$, as desired. 
Next we verify the description of the socle of $\Lambda$. Note that, for any $\Lambda$ module $M$, we have $\mathfrak{m} M=0$ if and only if $M$ is (a $k$-module hence) a semisimple $\Lambda$-module. Therefore $\mathfrak{m}^{3}=0$ implies that $\mathfrak{m}^{2} \subseteq \operatorname{soc} \Lambda$. Conversely, take $c \in \operatorname{soc} \Lambda$ and write $c$ in the standard form of Lemma 4.2. Then $0=c x=u_{2} x y$ shows that $u_{2}$ is not a unit, and hence $u_{2}=0$. But then $0=c y=u_{1} x y$, and so $u_{1}=0$, completing the proof that $\mathfrak{m}^{2}=\operatorname{soc} \Lambda=\Lambda x y+\Lambda y^{2}$. The sum $\Lambda x y+\Lambda y^{2}$ must be direct since $\mu_{\Lambda}\left(\mathfrak{m}^{2}\right)=2$. This completes the proof of the assertions about $\Lambda$.

$\Lambda 1_{x y}$. The second intersection in (iii) holds since $\Lambda x \cap \Lambda y=\Lambda x y$. The monomial $x_{x y}$ is nonzero since otherwise $x \in \Lambda x y$ contradicting $\mu_{\Lambda}(x, y)=2$. Similarly $y_{x y} \neq 0$; and $y_{x y}^{2} \neq 0$ because $\mu_{\Lambda}\left(x y, y^{2}\right)=2$.

We have soc $\Lambda 1_{x y} \supseteq \Lambda x_{x y}+\Lambda y_{x y}^{2}$ because the right-hand side is annihilated by $x$ and $y$. For the opposite inclusion take $c \in \Lambda$ such that $c_{x y} \in \operatorname{soc} \Lambda 1_{x y}$. Since $c_{x y} \mathfrak{m}=0$ we have $c \mathfrak{m} \subseteq \Lambda x y$. Now write $c$ in the standard form of Lemma 4.2. Then $c y=u_{1} x y+u_{2} y^{2} \in \Lambda x y$. Since $c y \in \Lambda x y$, directness of the sum $\Lambda x y \oplus \Lambda y^{2}$ shows that $u_{2}$ cannot be a unit, hence equals zero. Therefore $c=u_{1} x+u_{3} x y+u_{4} y^{2}$, showing that $c_{x y} \in \Lambda x_{x y}+\Lambda y_{x y}^{2}$. It remains to prove directness of this last sum; and this follows from $\Lambda x_{x y} \cap \Lambda y_{x y}=0$, completing the proof of all assertions about $\Lambda 1_{x y}$.

$\Lambda 1_{y}$. All assertions here are obvious.

(iv). It suffices to prove that $c y_{x y}=0 \Longleftrightarrow c x=0$. Since $c$ is not a unit, we can write it in the standard form of Lemma 4.2. Then $c y_{x y}=0$ is equivalent to $u_{1} x y+u_{2} y^{2} \in \Lambda x y$. Directness of the sum in our expression for soc $\Lambda$ shows that $u_{2}$ cannot be a unit, hence equals 0 . Therefore $c y_{x y}=0$ implies that $c=u_{1} x+u_{3} x y+u_{4} y^{2}$, and therefore $c x=0$. The converse is proved similarly.

Definition $4.4\left(\mathcal{S}\left(1 \frac{1}{2}\right), \mathcal{S}(2)\right)$. We define an object of the category $\mathcal{S}\left(1 \frac{1}{2}\right)$, or more completely, $k-\mathcal{S}\left(1 \frac{1}{2}\right)$ ("one and one-half $k$-similarity") to be an ordered triple $(m, n, \phi)$ where $m \leq n$ are positive integers and $\phi$ is an $n \times n$ matrix over $k$. We define a morphism of $\mathcal{S}\left(1 \frac{1}{2}\right)$ to be a pair of $k$-linear maps $(\sigma, \tau)$ (equivalently, matrices of the appropriate sizes) such that the following diagram commutes.

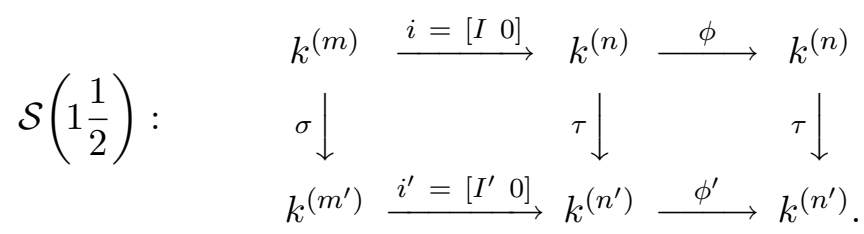

Here $k^{(m)}$ denotes the direct sum of $m$ copies of $k$, written as row vectors, and $i=i_{m, n}$ denotes the inclusion map $\mathbf{b} \rightarrow(\mathbf{b}, \mathbf{0})$, where $\mathbf{b}$ denotes an arbitrary vector in $k^{(m)}$ and $\mathbf{0}$ denotes the zero vector in $k^{(n-m)}$. The map $i^{\prime}$ is defined analogously. 
Similarly, we define the the category $\mathcal{S}(2)$ (more completely, $k-\mathcal{S}(2)$ ) to be "simultaneous similarity" of a pair of matrices over the field $k$. That is, the objects of $\mathcal{S}(2)$ are ordered triples $(n, A, B)$ where $n$ is a positive integer and $A$ and $B$ are $n \times n$ matrices over $k$. We define a morphism of $\mathcal{S}(2)$ to be a $k$-linear map (equivalently, matrix of the appropriate size) $\tau$ such that the following diagram commutes.

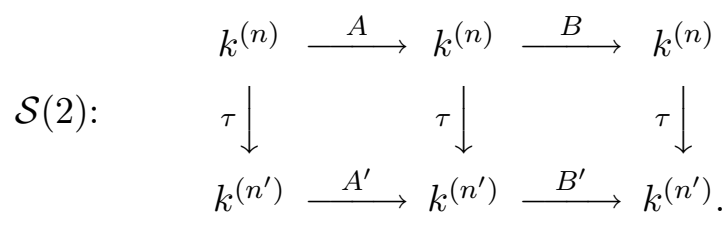

For the connection between these categories and wildness, first recall [Remarks 2.3(i)] that fdmod- $k\langle X, Y\rangle$ contains a copy of fdmod- $A$ for every finitely generated $k$-algebra $A$. The remaining (known) facts that we need are contained in the following lemma.

Lemma 4.5. Let $k$ be a field. Then:

(i) $\mathcal{S}(2)$ is equivalent to the category fdmod- $k\langle X, Y\rangle$ of finite dimensional right $k\langle X, Y\rangle$-modules.

(ii) $\mathcal{S}\left(1 \frac{1}{2}\right)$ contains a full subcategory that is equivalent to $\mathcal{S}(2)$.

(iii) Let $k$ be a residue field of a commutative ring $\Lambda$. Suppose that there is full subcategory $\mathcal{W}$ of the category of $\Lambda$-modules of finite length and an additive functor $\Phi: \mathcal{W} \rightarrow \mathcal{S}\left(1 \frac{1}{2}\right)$ that is a representation equivalence. Then $\Lambda$ is finite-length wild.

Proof. (i) It is easy to see that the category $\mathcal{S}(2)$ is equivalent to the category fdmod- $k\langle X, Y\rangle$ of finite dimensional right $k\langle X, Y\rangle$-modules by sending the object $(n, A, B)$ to the $k\langle X, Y\rangle$-module consisting of the vector space $k^{(n)}$, on which $X$ and $Y$ act via right multiplication by the matrices $A$ and $B$, respectively.

(ii) This is due to Narazova [ $\mathbf{N z}$, Lemma 1], except for a more functorial treatment here, and some changes and reversals of notation. For completeness, we include a proof.

Let $\mathcal{W}^{\prime}$ be the full subcategory of $\mathcal{S}\left(1 \frac{1}{2}\right)$ consisting of objects of the form $(n, 2 n, \phi)$ in which $\phi=\left[\begin{array}{cc}0 & I \\ A & B\end{array}\right]$, where $A, B$ are $n \times n$ matrices over $k$ and $0, I$ are the $n \times n$ zero and identity matrices, respectively. Then let $F(n, 2 n, \phi)=(n, A, B) \in \mathcal{S}(2)$.

Let $(\sigma, \tau):(n, 2 n, \phi) \rightarrow\left(n^{\prime}, 2 n^{\prime}, \pi^{\prime}\right)$ be a morphism in $\mathcal{W}^{\prime}$. We claim that $\tau$ has the $2 \times 2$ block-diagonal form $\tau=\left[\begin{array}{cc}\sigma & 0 \\ 0 & \sigma\end{array}\right]$. To see this, let $\tau=\left[\begin{array}{ll}T_{1} & T_{2} \\ T_{3} & T_{4}\end{array}\right]$. 
The relation $i \tau=\sigma i^{\prime}$ (as right operators) yields the equation

$$
\left[\begin{array}{ll}
I & 0
\end{array}\right]\left[\begin{array}{ll}
T_{1} & T_{2} \\
T_{3} & T_{4}
\end{array}\right]=\left[\begin{array}{ll}
\sigma & 0
\end{array}\right]
$$

which shows that $T_{1}=\sigma$ and $T_{2}=0$. Then the relation $\tau \phi^{\prime}=\phi \tau$ yields the equation

$$
\left[\begin{array}{cc}
T_{1} & 0 \\
T_{3} & T_{4}
\end{array}\right]\left[\begin{array}{cc}
0 & I \\
A^{\prime} & B^{\prime}
\end{array}\right]=\left[\begin{array}{cc}
0 & I \\
A & B
\end{array}\right]\left[\begin{array}{cc}
T_{1} & 0 \\
T_{3} & T_{4}
\end{array}\right]
$$

which easily completes the proof of the claim. Moreover, the relation $\tau \phi^{\prime}=$ $\phi \tau$ now implies that $\sigma A^{\prime}=A \sigma$ and $\sigma B^{\prime}=B \sigma$, and hence these left and right multiplications by $\sigma$ form a morphism $F(\sigma, \tau)=\sigma$ in $\mathcal{S}(2)$.

The block diagonal form of $\tau$ together with the relations in the previous paragraph then show that $(\sigma, \tau)$ is an isomorphism if and only if $\sigma$ is an invertible matrix if and only if $F(\sigma, \tau)=\sigma$ is an isomorphism in $\mathcal{S}(2)$. It is now easy to check that $F$ yields an equivalence between $\mathcal{W}^{\prime}$ and $\mathcal{S}(2)$.

(iii) In view of statements (i) and (ii), this is an immediate consequence of the definition [2.2] of finite-length wildness

Definition 4.6 (Ringel's $\Lambda$-module $M(m, n, \phi))$. Let $(\Lambda, \mathfrak{m}, k)$ be a Drozd ring; given an object $(m, n, \phi) \in \mathcal{S}\left(1 \frac{1}{2}\right)$, we define a module $M(m, n, \phi)$ as follows. First, let $Q$ be the $\Lambda$-module $Q=\Lambda^{(m)} 1_{y} \oplus \Lambda^{(n)} 1_{x y} \oplus \Lambda^{(n)}$ (notation as in (4.3.1)), where $\Lambda^{(m)}$ denotes the direct sum of $m$ copies of the $\Lambda$-module $\Lambda$, written as row vectors. Next, define three maps $i$, mult, and $\phi$ between pairs of submodules of $Q$, as follows. [See diagram (4.6.1).]

The map $i: \Lambda^{(m)} x_{y} \rightarrow \Lambda^{(n)} y_{x y}^{2}$. Since the socle of every $\Lambda$-module is annihilated by $\mathfrak{m}$, we have $\Lambda^{(m)} x_{y}=k^{(m)}$; more precisely, this is a canonical $\Lambda$-module isomorphism via $\mathbf{b} x_{y} \rightarrow \overline{\mathbf{b}}$ (where $\overline{\mathbf{b}}$ denotes the vector in $k^{(m)}$ formed by reducing each entry of $\mathbf{b}$ modulo $\mathfrak{m})$. Similarly $\Lambda^{(n)} y_{x y}^{2}=k^{(n)}$ via $\mathbf{c} y_{x y}^{2} \rightarrow \overline{\mathbf{c}}$. We define $i=i_{m, n}$ by $\left(\mathbf{b} x_{y}\right) i=(\mathbf{b}, \mathbf{0}) y_{x y}^{2}$, the same map $i$ as in (4.4.1).

The map mult: $\Lambda^{(n)} x \rightarrow \Lambda^{(n)} y_{x y}$. This is by means of the multiplication map $\mathbf{d} x \rightarrow \mathbf{d} y_{x y}$. By Lemma 4.3(iv) this is indeed an $\Lambda$-module isomorphism.

The map $\phi: \Lambda^{(n)} x_{x y} \rightarrow \Lambda^{(n)} y^{2}$. We have $\Lambda^{(n)} x_{x y}=k^{(n)}$ via $\mathbf{c} x_{x y} \rightarrow \overline{\mathbf{c}}$. Therefore we can right-multiply elements of $\Lambda^{(n)} x_{x y}=k^{(n)}$ by the matrix $\phi$ in our given object $(m, n, \phi)$ of $\mathcal{S}\left(1 \frac{1}{2}\right)$. Also, $\Lambda^{(n)} y^{2}=k^{(n)}$ via $\mathbf{c} y^{2} \rightarrow \overline{\mathbf{c}}$. For $\mathbf{c} \in \Lambda^{(n)}$ define $\left(\mathbf{c} x_{x y}\right) \phi=(\overline{\mathbf{c}} \phi) y^{2}$.

These three maps are displayed in the following diagram. 
$(4.6 .1)$

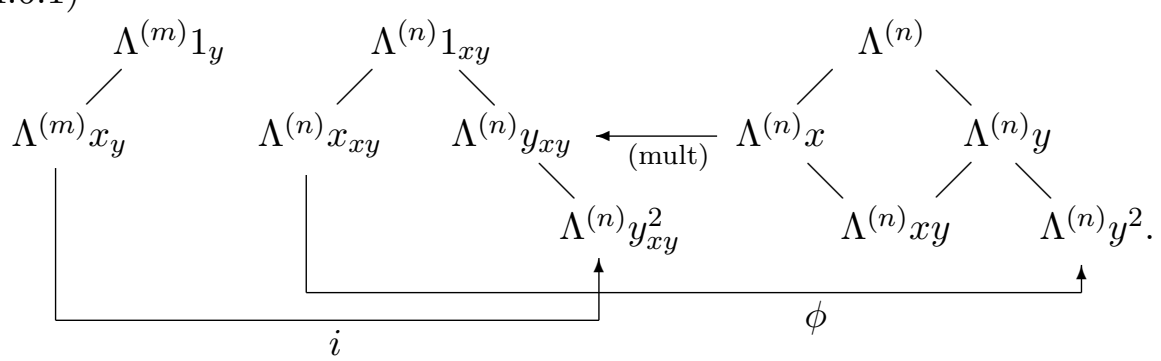

Finally, we define the $\Lambda$-module $M=M(m, n, \phi)$ to be $Q / \mathcal{R}$ where $\mathcal{R}$ denotes the submodule formed by the following set of relations, that is, elements of $Q$.

$$
\left(\mathbf{b} x_{y},-(\mathbf{b} i) y_{x y}^{2}, \mathbf{0}\right)+\left(\mathbf{0},-\mathbf{d} y_{x y}, \mathbf{d} x\right)+\left(\mathbf{0}, \mathbf{c} x_{x y},-(\overline{\mathbf{c}} \phi) y^{2}\right)
$$

where $\mathbf{b}, \mathbf{c}, \mathbf{d}$ range over all elements of $\Lambda^{(m)}, \Lambda^{(n)}, \Lambda^{(n)}$ respectively. (Note that we can replace $\mathbf{b}, \mathbf{c}, \mathbf{d}$ by $\overline{\mathbf{b}}, \overline{\mathbf{c}}, \overline{\mathbf{d}}$ respectively, whenever convenient, but we cannot replace $\overline{\mathbf{c}}$ by $\mathbf{c}$ in $\overline{\mathbf{c}} \phi$.)

Informally, we form $M$ from $Q$ by identifying elements of $Q$ with their image under the above "amalgamation" maps $i$, mult, and $\phi$. (It is possible that $\phi$ have a nontrivial kernel, in which case it is not a true amalgamation map.)

For every element $(m, n, \phi) \in \mathcal{S}\left(1 \frac{1}{2}\right)$ we have natural $\Lambda$-module surjections:

$$
\rho: P=\Lambda^{(m)} \oplus \Lambda^{(n)} \oplus \Lambda^{(n)} \stackrel{\rho_{1}}{\longrightarrow} Q=\Lambda^{(m)} 1_{y} \oplus \Lambda^{(n)} 1_{x y} \oplus \Lambda^{(n)} \stackrel{\rho_{2}}{\longrightarrow} M(m, n, \phi)
$$

where $(\mathbf{b}, \mathbf{c}, \mathbf{d}) \rho_{1}=\left(\mathbf{b}_{y}, \mathbf{c}_{x y}, \mathbf{d}\right)$ and $\rho_{2}$ equals reduction modulo the relations $\mathcal{R}$ displayed in (4.6.2). Note that $\operatorname{ker} \rho \subseteq \mathfrak{m} P$, and therefore:

$$
\text { The map } \rho \text { in (4.6.3) is a projective cover. }
$$

Historical Remark. In Drozd's original paper $[\mathbf{D}], \Lambda$ is a $k$-algebra and the modules he uses in place of our $M$ are represented by very large matrices $(32 n \times 32 n$, in the notation of 4.4$)$ over $k$. He assumes that $k$ is an algebraically closed field. But, in order to obtain wildness of $\Lambda$, the only property of algebraic closure that he uses is that $k$ has at least 5 elements. (Algebraic closure is, however, used elsewhere in his paper.) Ringel $[\mathbf{R},(3.4)]$ uses much smaller matrices over $k$, by relating $M$ to $\mathcal{S}\left(1 \frac{1}{2}\right)$ rather than directly to $\mathcal{S}(2)$, as Drozd did. The size of Ringel's matrices is $(m+6 n) \times(m+6 n)$, in the notation of 4.4. Wildness of the category of $\Lambda$-modules that he obtains holds for any field $k$. 
Our module $M=M(m, n, \phi)$ is a modification of Ringel's construction, and follows him by relating $\Lambda$-modules to $\mathcal{S}\left(1 \frac{1}{2}\right)$ rather than directly to $\mathcal{S}(2)$. However, since our ring $\Lambda$ need not be a $k$-algebra, $M$ need not have a matrix representation. But we do represent homomorphisms in the category of modules $M(m, n, \phi)$ by matrices over the ring $\Lambda$. The critical fact is that any map of $\Lambda$-modules lifts to a map of their projective covers; and since projective modules over commutative local rings are free, this lifted map can be represented by a matrix over $\Lambda$. Our $\Lambda$-matrices are much smaller than Ringel's $k$-matrices. But this is deceptive because an element of $\Lambda$ holds more information than a single element of $k$. There are two consequences of this: (i) The functor that we obtain, displaying wildness, is a only a representation equivalence rather than the full exact embedding that Ringel gets. (ii) Our wildness proof is probably more intricate but shorter than Ringel's (omitted) proof.

Morphisms and Matrices. Let $f: M=M(m, n, \phi) \rightarrow M^{\prime}=M\left(m^{\prime}, n^{\prime}, \phi^{\prime}\right)$ be any $\Lambda$-module homomorphism. We wish to represent $f$ by a matrix $F$ over $\Lambda$. Since $P$ is a projective $\Lambda$-module, $f$ lifts to a $\Lambda$-homomorphism such that the following diagram commutes.

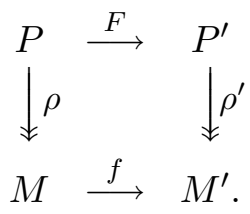

Any such $F$ equals right multiplication by a unique $(m+2 n) \times\left(m^{\prime}+2 n^{\prime}\right)$ matrix over $\Lambda$, and we again call this matrix $F$.

Block form. We always view $F$ in $3 \times 3$ block form, where the rows are partitioned into three blocks of lengths $m, n$, and $n$, respectively, and the columns are partitioned into three blocks of lengths $m^{\prime}, n^{\prime}$, and $n^{\prime}$, respectively.

The crux of our wildness proof is the next lemma, which gives some information about the form of the matrix $F$.

Notation 4.7. We denote by ent $\mathbf{e}$ the set of entries of any array $\mathbf{e}$.

\section{Lemma 4.8.}

(i) The matrix $F$ has the block form

$$
F=\left[\begin{array}{ccc}
F_{11} & \mathfrak{m} & \left(x, y^{2}\right) \\
* & F_{22} & \mathfrak{m} \\
* & * & F_{33}
\end{array}\right] .
$$

That is, ent $F_{12} \subseteq \mathfrak{m}$, ent $F_{23} \subseteq \mathfrak{m}$, and ent $F_{13} \subseteq\left(x, y^{2}\right)$. Moreover, when all entries are reduced modulo $\mathfrak{m}$, the diagonal blocks satisfy the following three properties (viewed as right operators). 
(a) $\bar{F}_{11} i_{m^{\prime}, n^{\prime}}=i_{m, n} \bar{F}_{22}$, where $i_{m^{\prime}, n^{\prime}}$ and $i_{m, n}$ are as in (4.4.1).

(b) $\bar{F}_{22}=\bar{F}_{33}$.

(c) $\phi \bar{F}_{22}=\bar{F}_{22} \phi^{\prime}$.

(We make no assertion about the blocks denoted by *.)

(ii) If $f$ is an isomorphism, then the diagonal blocks $F_{i i}$ are invertible matrices.

Proof. Let $b, c, d$ be elements of a local ring with residue field $k$ (e.g., $\Lambda$ or its homomorphic images).

$$
\text { If } b d=c d \text { and } d \neq 0 \text { then } \bar{b}=\bar{c} \text { (natural images in } k \text { ) }
$$

because $b \equiv c$ modulo the annihilator of $d$, and this annihilator is contained in the maximal ideal of the ring. We use this fact many times in the long, intricate proof below.

(i) Since $F$ lifts $f$ we must have $(\operatorname{ker} \rho) F \subseteq \operatorname{ker} \rho^{\prime}$. Recall that we factor $\rho=\rho_{1} \rho_{2}$ as in (4.6.3). The information that we obtain about $F$ is information that can be obtained by dealing separately with five types of elements of $\operatorname{ker} \rho$. Type 1: Elements $(\mathbf{e}, \mathbf{0}, \mathbf{0}) \in P$ such that $(\mathbf{e}, \mathbf{0}, \mathbf{0}) \rho_{1}=\mathbf{0} \in Q$; type 2: Elements $(\mathbf{0}, \mathbf{e}, \mathbf{0}) \in P$ such that $(\mathbf{0}, \mathbf{e}, \mathbf{0}) \rho_{1}=\mathbf{0}$ in $Q$; types $3-5$ : Elements of $P$ corresponding to the three types of amalgamations that were used to form $M$, one type for each term in (4.6.2). We label the five parts of the proof by the blocks of $F$ about which they give information.

$F_{12}, F_{13}$. Note that $(\mathbf{e} y, \mathbf{0}, \mathbf{0}) \rho_{1}=\mathbf{0}$ for every e because $y_{y}=0$. Therefore $(\mathbf{e} y, \mathbf{0}, \mathbf{0}) F \rho^{\prime}=0$ and hence $(\mathbf{e} y, \mathbf{0}, \mathbf{0}) F \rho_{1}^{\prime} \subseteq \operatorname{ker} \rho_{2}^{\prime}$. Writing $F$ in block form and using the definition of $\rho_{1}^{\prime}$, it follows that

$$
\left(\mathbf{0}, \mathbf{e} F_{12} y_{x y}, \mathbf{e} F_{13} y\right) \in \operatorname{ker} \rho_{2}^{\prime}
$$

for every e. Therefore every element of this form must have an expression of the form (4.6.2) (more precisely, the version of (4.6.2) that applies to $M^{\prime}$, hence contains $i^{\prime}$ and $\phi^{\prime}$ in place of $i$ and $\left.\phi\right)$.

Comparing coordinate 1 in (4.6.2) and (4.8.3) yields ent $\mathbf{b} \subseteq \mathfrak{m}$. Therefore the first of the three terms in (4.6.2) equals zero, and can be ignored. Looking at coordinate 3 shows that

$$
\mathbf{e} F_{13} y=\mathbf{d} x-(\overline{\mathbf{c}} \phi) y^{2} .
$$

Therefore ent $\mathbf{d} \subseteq \mathfrak{m}$ (otherwise $x \in \Lambda y$ and $\mu_{\Lambda}(\mathfrak{m}) \leq 1$ ). On the other hand, looking at coordinate 2 shows that $\mathbf{e} F_{12} y_{x y}=-\mathbf{d} y_{x y}+\mathbf{c} x_{x y}$. But $\Lambda x_{x y} \cap \Lambda y_{x y}=0$ by (4.3.1). Therefore ent $\mathbf{c} \subseteq \mathfrak{m}$. A second look at coordinate 2 therefore shows that $\operatorname{ent}\left(\mathbf{e} F_{12} y_{x y}\right) \subseteq \mathfrak{m}^{2}$ for every e. Hence ent $F_{12} \subseteq \mathfrak{m}$ as desired.

Since ent $\mathbf{c} \subseteq \mathfrak{m}$, we have $\overline{\mathbf{c}}=\mathbf{0}$. But ent $\mathbf{d} \subseteq \mathfrak{m}$ also, so (4.8.4) now yields

$$
\text { ent } \mathbf{e} F_{13} y \subseteq \text { ent } \mathbf{d} x \subseteq \mathfrak{m} x=\Lambda x y
$$


for every e. Let $c$ be any element of ent $F_{13}$. Then $c y \in \Lambda x y$, so that $c \in \mathfrak{m}$. Write $c$ in the standard form of Lemma 4.2. Then

$$
c y=u_{1} x y+u_{2} y^{2} \in \Lambda x y .
$$

Directness of the sum $\Lambda x y+\Lambda y^{2}$ then shows that $u_{2}$ is not a unit, and hence $u_{2}=0$. Therefore $c \in\left(x, y^{2}\right)$ and hence ent $F_{13} \subseteq\left(x, y^{2}\right)$ as desired.

$F_{23}$. Note that $(\mathbf{0}, \mathbf{e} x y, \mathbf{0}) \rho_{1}=\mathbf{0}$ for every e because $(x y)_{x y}=0$. Therefore, as before, $(\mathbf{0}, \mathbf{e} x y, \mathbf{0}) F \rho_{1}^{\prime} \subseteq \operatorname{ker} \rho_{2}^{\prime}$ and hence

$$
\left(\mathbf{0}, \mathbf{0}, \mathbf{e} F_{23} x y\right) \in \operatorname{ker} \rho_{2}^{\prime}
$$

for every e. As before, this triple must have the form (4.6.2). Looking at coordinate 1 in both triples shows that ent $\mathbf{b} \subseteq \mathfrak{m}$, hence term 1 of (4.6.2) equals zero and we can ignore it. Coordinate 2 then yields $-\mathbf{d} y_{x y}+\mathbf{c} x_{x y}=\mathbf{0}$. Since $\Lambda x_{x y} \cap \Lambda y_{x y}=0$ we have

$$
\mathbf{c} x_{x y}=\mathbf{0} \quad \text { and } \quad \mathbf{d} y_{x y}=\mathbf{0} .
$$

In particular, ent $\mathbf{c} \subseteq \mathfrak{m}$ and ent $\mathbf{d} \in \mathfrak{m}$. Write an arbitrary entry $d$ of $\mathbf{d}$ in standard form $d=u_{1} x+u_{2} y+u_{3} x y+u_{4} y^{2}$. Then (4.8.6) yields $0=d y_{x y}=u_{2} y_{x y}^{2}$. Therefore $u_{2}$ is not a unit, and hence $u_{2}=0$. We conclude that $d \in\left(x, y^{2}\right)$. That is, ent $\mathbf{d} \subseteq\left(x, y^{2}\right)$, from which it follows that $\mathbf{d} x=\mathbf{0}$. Since ent $\mathbf{c} \subseteq \mathfrak{m}$ we have $\overline{\mathbf{c}}=\mathbf{0}$ and hence coordinate 3 of (4.6.2) equals $\mathbf{0}$. Therefore (4.8.5) yields $\mathbf{e} F_{23} x y=\mathbf{0}$ for every $\mathbf{e}$. This, in turn, implies that ent $F_{23} \subseteq \mathfrak{m}$, as desired.

$\bar{F}_{11} i_{m^{\prime}, n^{\prime}}=i_{m, n} \bar{F}_{22}$. The first amalgamation term in (4.6.2) is carried by $\rho$ to zero: $\left(\mathbf{e} x,-\left(\mathbf{e} i_{m, n}\right) y^{2}, \mathbf{0}\right) \rho=0$ for every $\mathbf{e}$. Therefore

$$
\left(\mathbf{e} x,-\left(\mathbf{e} i_{m, n}\right) y^{2}, \mathbf{0}\right) F \rho_{1}^{\prime} \in \operatorname{ker} \rho_{2}^{\prime} .
$$

Writing $F$ in block form and using the definition of $\rho_{2}^{\prime}$ then shows

$$
\begin{aligned}
&\left(\mathbf{e} F_{11} x_{y}-\left(\mathbf{e} i_{m, n}\right) F_{21} y_{y}^{2}, \mathbf{e} F_{12} x_{x y}-\left(\mathbf{e} i_{m, n}\right) F_{22} y_{x y}^{2}\right. \\
&\left.\mathbf{e} F_{13} x-\left(\mathbf{e} i_{m, n}\right) F_{23} y^{2}\right) \in \operatorname{ker} \rho_{2}^{\prime} .
\end{aligned}
$$

Since $y_{y}=0, F_{12} x_{x y} \in \mathfrak{m} x_{x y}=0$, ent $F_{13} \subseteq\left(x, y^{2}\right)$, and ent $F_{23} \subseteq \mathfrak{m}$ this simplifies to

$$
\left(\mathbf{e} F_{11} x_{y},-\left(\mathbf{e} i_{m, n}\right) F_{22} y_{x y}^{2}, \mathbf{0}\right) \in \operatorname{ker} \rho_{2}^{\prime} .
$$

We have $\Lambda x \cap \Lambda y^{2}=\Lambda x \cap \Lambda y \cap \Lambda y^{2}=\Lambda x y \cap \Lambda y^{2}=0$ by (4.3.1). Comparing coordinate 3 of (4.8.7) with coordinate 3 of (4.6.2) yields $\mathbf{d} x-(\overline{\mathbf{c}} \phi) y^{2}=\mathbf{0}$; therefore $\mathbf{d} x=\mathbf{0}$, and hence ent $\mathbf{d} \subseteq \mathfrak{m}$. Then writing a typical entry of $\mathbf{d}$ in standard form of Lemma 4.2, together with the relation $\mathbf{d} x=\mathbf{0}$, shows that ent $\mathbf{d} \subseteq\left(x, y^{2}\right)$. Therefore term 2 in (4.6.2) equals zero.

Coordinate 2 of (4.8.7) and (4.6.2) now yields

$$
-\left(\mathbf{e} i_{m, n}\right) F_{22} y_{x y}^{2}=-\left(\mathbf{b} i_{m^{\prime}, n^{\prime}}\right) y_{x y}^{2}+\mathbf{c} x_{x y} \text {. }
$$


Then $\Lambda x_{x y} \cap \Lambda y_{x y}^{2}=0$ yields

$$
\left(\mathbf{e} i_{m, n}\right) F_{22} y_{x y}^{2}=\left(\mathbf{b} i_{m^{\prime}, n^{\prime}}\right) y_{x y}^{2} .
$$

Coordinate 1 yields $\mathbf{e} F_{11} x_{y}=\mathbf{b} x_{y}$. Then (4.8.2) yields $\overline{\mathbf{e}} \bar{F}_{11}=\overline{\mathbf{b}}$ and hence $\overline{\mathbf{e}} \bar{F}_{11} i_{m^{\prime}, n^{\prime}}=\overline{\mathbf{b}} i_{m^{\prime}, n^{\prime}}$. But (4.8.8) yields $\left(\overline{\mathbf{e}} i_{m, n}\right) \bar{F}_{22}=\overline{\mathbf{b}} i_{m^{\prime}, n^{\prime}}$ (also by (4.8.2)). We conclude that $\overline{\mathbf{e}} \bar{F}_{11} i_{m^{\prime}, n^{\prime}}=\left(\overline{\mathbf{e}} i_{m, n}\right) \bar{F}_{22}$ for every e, and therefore $\bar{F}_{11} i_{m^{\prime}, n^{\prime}}=i_{m, n} \bar{F}_{22}$, proving (a).

$\bar{F}_{22}=\bar{F}_{33}$. We use the second amalgamation term in (4.6.2): $(\mathbf{0},-\mathbf{e} y, \mathbf{e} x) \rho$ $=0$ and therefore $(\mathbf{0},-\mathbf{e} y, \mathbf{e} x) F \rho_{1}^{\prime} \subseteq \operatorname{ker} \rho_{2}^{\prime}$ for every e. Writing $F$ in block form and applying the definition of $\rho_{1}^{\prime}$ yields

$$
\left(\ldots,-\mathbf{e} F_{22} y_{x y}+\mathbf{e} F_{32} x_{x y},-\mathbf{e} F_{23} y+\mathbf{e} F_{33} x\right) \in \operatorname{ker} \rho_{2}^{\prime} .
$$

Comparing coordinate 2 in this and (4.6.2) and multiplying by $y$ yields $\mathbf{e} F_{22} y_{x y}^{2}=\mathbf{d} y_{x y}^{2}$. Then (4.8.2) yields $\overline{\mathbf{e}} \bar{F}_{22}=\overline{\mathbf{d}}$.

Comparing coordinate 3 and multiplying by $y$ yields $-\mathbf{e} F_{23} y^{2}+\mathbf{e} F_{33} x y=$ $\mathbf{d} x y$. Since $\Lambda x y \cap \Lambda y^{2}=0$, this yields $\overline{\mathbf{e}} \bar{F}_{33}=\overline{\mathbf{d}}$ (by (4.8.2)). Therefore $\overline{\mathbf{e}} \bar{F}_{22}=\overline{\mathbf{e}} \bar{F}_{33}$ for every e, and hence $\bar{F}_{22}=\bar{F}_{33}$, proving (b).

$\phi \bar{F}_{22}=\bar{F}_{22} \phi^{\prime}$. Use the third amalgamation term in (4.6.2): (0, ex, $\left.-(\overline{\mathbf{e}} \phi) y^{2}\right) \rho=0$ and hence $\left(\mathbf{0}, \mathbf{e} x,-(\overline{\mathbf{e}} \phi) y^{2}\right) F \rho_{1}^{\prime} \subseteq \operatorname{ker} \rho_{2}^{\prime}$. As before this yields

$$
\left(\ldots, \mathbf{e} F_{22} x_{x y}-(\overline{\mathbf{e}} \phi) F_{32} y_{x y}^{2}, \mathbf{e} F_{23} x-(\overline{\mathbf{e}} \phi) F_{33} y^{2}\right) \subseteq \operatorname{ker} \rho_{2}^{\prime}
$$

and this therefore has the form (4.6.2) for all e. Comparing coordinate 2 in these expressions yields

$$
\mathbf{e} F_{22} x_{x y}-(\overline{\mathbf{e}} \phi) F_{32} y_{x y}^{2}=-\left(\mathbf{b} i^{\prime}\right) y_{x y}^{2}-\mathbf{d} y_{x y}+\mathbf{c} x_{x y} .
$$

Since $\Lambda x_{x y} \cap \Lambda y_{x y}=0$, comparing the $x_{x y}$-terms on both sides yields $\mathbf{e} F_{22} x_{x y}=\mathbf{c} x_{x y}$, and therefore $\overline{\mathbf{e}} \bar{F}_{22}=\overline{\mathbf{c}}$ (by (4.8.2)). We conclude that

$$
\overline{\mathbf{e}} \bar{F}_{22} \phi^{\prime}=\overline{\mathbf{c}} \phi^{\prime}
$$

for all e.

Comparing coordinate 3 in (4.8.10) and (4.6.2) yields

$$
\mathbf{e} F_{23} x-(\overline{\mathbf{e}} \phi) F_{33} y^{2}=\mathbf{d} x-\left(\overline{\mathbf{c}} \phi^{\prime}\right) y^{2} .
$$

As already observed, $\Lambda x \cap \Lambda y^{2}=0$. Therefore $(\overline{\mathbf{e}} \phi) F_{33} y^{2}=\left(\overline{\mathbf{c}} \phi^{\prime}\right) y^{2}$, so that by (4.8.2) we get $(\overline{\mathbf{e}} \phi) \bar{F}_{33}=\overline{\mathbf{c}} \phi^{\prime}$. Comparing this with (4.8.12) yields $(\overline{\mathbf{e}} \phi) \bar{F}_{33}=\overline{\mathbf{e}} \bar{F}_{22} \phi^{\prime}$ for every e, and hence $\phi \bar{F}_{33}=\bar{F}_{22} \phi^{\prime}$. Since $\bar{F}_{33}=\bar{F}_{22}$, statement (c) follows, and the proof of part (i) of the lemma is now complete.

(ii) We assume that $f$ is an isomorphism. Since $\rho$ and $\rho^{\prime}$ are projective covers (see (4.6.4)), the map $F$ is again an isomorphism, and therefore the matrix $F$ is invertible. Hence $\bar{F}$ is invertible. But $\bar{F}$ is a block-triangular 
matrix over the field $k$, by part (i) of this proof, and hence each diagonal block $\bar{F}_{i i}$ is invertible. Therefore $F_{i i}$ is also invertible.

Theorem 4.9 (Wildness of Drozd Rings). Every Drozd ring is finite-length wild.

Proof. Let the Drozd ring be $(\Lambda, \mathfrak{m}, k)$. Since the $\Lambda$-modules $M(m, n, \phi)$ have finite length, by Lemma 4.5(iii) it suffices to show:

(4.9.1) Let $\mathcal{W}$ the full subcategory of $\bmod \Lambda$ consisting of all modules of the form $M(m, n, \phi)$. Then there is an additive functor $\mathcal{E}: \mathcal{W} \rightarrow \mathcal{S}\left(1 \frac{1}{2}\right)$ that is a representation equivalence.

For each $M=M(m, n, \phi) \in \mathcal{W}$ fix a pair of surjective homomorphisms $\rho_{1}: P=\Lambda^{(m)} \oplus \Lambda^{(n)} \oplus \Lambda^{(n)} \rightarrow Q$ and $\rho_{2}: Q \rightarrow M$, and let $\rho$ be their composition as in (4.6.3). In particular, $\operatorname{ker}\left(\rho_{2}\right)$ is given by (4.6.2), and the matrix $\phi$ acts as shown in (4.6.1). These choices remain in effect throughout this proof, and our definition of $\mathcal{E}$ depends on them. In particular, set $\mathcal{E}(M)=(m, n, \phi) \in \mathcal{S}\left(1 \frac{1}{2}\right)$.

Next let $f: M \rightarrow M^{\prime}$ be a $\Lambda$-homomorphism, where $M=M(m, n, \phi)$ and $M^{\prime}=M\left(m^{\prime}, n^{\prime}, \phi^{\prime}\right)$. We wish to define the morphism $\mathcal{E}(f)=(\sigma, \tau)$; that is, we wish to define $\sigma$ and $\tau$ in diagram (4.4.1).

Recall that $\rho: P \rightarrow M$ and $\rho^{\prime}: P^{\prime} \rightarrow M^{\prime}$ are projective covers. Therefore $f$ lifts to a homomorphism $F: P \rightarrow P^{\prime}$ such that diagram (4.6.5) commutes. The matrix of $F$ (again called $F$ ) has a $3 \times 3$ block upper triangular form, as displayed in (4.8.1). Then we define

$$
\mathcal{E}(f)=\left(\bar{F}_{11}, \bar{F}_{22}\right) \text {. }
$$

We proceed to prove that $\mathcal{E}$ has the required properties.

First we claim that $\mathcal{E}(f)$ is well-defined; that is, choosing a different lifting $F$ of $f$ does not change $\mathcal{E}(f)$. It suffices to show that if $f=0$ then $\bar{F}_{11}$ and $\bar{F}_{22}$ equal 0 . But $f=0$ implies that $F(P) \subseteq \operatorname{ker} \rho^{\prime} \subseteq \mathfrak{m} P^{\prime}$, and hence all entries of the matrix $F$ are elements of $\mathfrak{m}$. Hence the entire matrix $\bar{F}$ equals 0 , proving the claim.

Next, we claim that $\mathcal{E}(f)$ is a morphism in $\mathcal{S}\left(1 \frac{1}{2}\right)$, that is, that diagram (4.4.1) commutes. For that, we must show that, for every $\mathbf{b} \in \Lambda^{(m)}$ and $\mathbf{c} \in$ $\Lambda^{(n)}$, both $\overline{\mathbf{b}} i \bar{F}_{22}=\overline{\mathbf{b}} \bar{F}_{11} i^{\prime}$ and $\overline{\mathbf{c}} \phi \bar{F}_{22}=\overline{\mathbf{c}} \bar{F}_{22} \phi^{\prime}$. But these two equations follow immediately from parts (i)(a) and (i)(c) of Lemma 4.8.

Since $\mathcal{E}$ is well-defined on morphisms, it is now easy to complete the proof that $\mathcal{E}$ is an additive functor. For example the identity map on a module can be lifted to the identity map on its projective cover and then again becomes an identity map modulo $\mathfrak{m}$, showing that $\mathcal{E}(1)=1$. Similarly $\mathcal{E}$ preserves compositions and sums of morphisms.

We now check the three defining properties of a representation equivalence. 
Dense, that is, $\mathcal{E}$ maps onto all isomorphism classes.

This is clear from the definition of $\mathcal{W}$ as the full subcategory of $\bmod \Lambda$ consisting of all modules of the form $M(m, n, \phi)$.

Full, that is, $\mathcal{E}$ is a surjection on hom groups.

Let $(\sigma, \tau)$ be a morphism in $\mathcal{S}\left(1 \frac{1}{2}\right)$, as displayed in (4.4.1). Each of $\sigma, \tau$ equals right multiplication by a $k$-matrix that we again call $\sigma, \tau$ respectively. Let $F$ be a block diagonal matrix over $\Lambda$ such that $\bar{F}_{11}=\sigma, \bar{F}_{22}=\tau$, $F_{33}=F_{22}$, and all other entries of $F$ are zero. Right multiplication by $F$ is a homomorphism of free $\Lambda$-modules $P \rightarrow P^{\prime}$. To show that $F$ induces an $\Lambda$-homomorphism $M \rightarrow M^{\prime}$ it suffices to check that $(\operatorname{ker} \rho) F \subseteq \operatorname{ker} \rho^{\prime}$. The block diagonal form of $F$ shows that $F$ induces an $\Lambda$-module homomorphism $F^{\prime}: Q=P \rho_{1} \rightarrow Q^{\prime}=P^{\prime} \rho_{1}^{\prime}$. Thus it now suffices to check that the induced map $F^{\prime}$ induces a homomorphism $M \rightarrow M^{\prime}$. For this, it suffices to check that $F^{\prime}$ takes each of the three terms of (4.6.2) to terms of the same form with respect to $M^{\prime}$. First we have

$$
\left(\mathbf{b} x_{y},-(\mathbf{b} i) y_{x y}, \mathbf{0}\right)\left(F_{11} \oplus F_{22} \oplus F_{33}\right)=\left(\mathbf{b} F_{11} x_{y},-(\mathbf{b} i) F_{22} y_{x y}, \mathbf{0}\right) .
$$

Since we are working in socles of $\Lambda$-modules, we can replace $F_{11}$ and $F_{22}$ by their images mod $\mathfrak{m}$. Moreover, since $\left(\bar{F}_{11}, \bar{F}_{22}\right)=(\sigma, \tau)$ is a morphism in $\mathcal{S}\left(1 \frac{1}{2}\right)$, we have $i \bar{F}_{22}=\bar{F}_{11} i^{\prime}$, and this shows that the form of $\left(\mathbf{b} x_{y},-(\mathbf{b} i) y_{x y}, \mathbf{0}\right)$ is preserved.

The form of the second term in (4.6.2) is preserved because $F_{22}=F_{33}$. For the third term we have

$$
\left(\mathbf{0}, \mathbf{c} x_{x y},-(\overline{\mathbf{c}} \phi) y^{2}\right)\left(F_{11} \oplus F_{22} \oplus F_{33}\right)=\left(\mathbf{0}, \mathbf{c} F_{22} x_{x y},-(\overline{\mathbf{c}} \phi) F_{33} y^{2}\right) .
$$

Since $x_{x y}$ and $y^{2}$ are in the socles of their respective modules, we can replace each of $F_{22}$ and $F_{33}$, on the right-hand side, by their respective images (both $\tau)$ modulo $\mathfrak{m}$. Since $\phi \tau=\tau \phi^{\prime}$ it is clear that the form of $\left(\mathbf{0}, \mathbf{c} x_{x y},-(\overline{\mathbf{c}} \phi) y^{2}\right)$ has been preserved.

Faithful, that is, $\mathcal{E}(M) \cong \mathcal{E}\left(M^{\prime}\right)$ if and only if $M \cong M^{\prime}$.

Since $\mathcal{E}$ is a functor, clearly $\mathcal{E}(M) \cong \mathcal{E}\left(M^{\prime}\right)$ if $M \cong M^{\prime}$.

For the converse, suppose that $(\sigma, \tau)$ is an isomorphism from $\mathcal{E}(M)$ to $\mathcal{E}\left(M^{\prime}\right)$ in the category $\mathcal{S}\left(1 \frac{1}{2}\right)$. Let $F$ be a block diagonal matrix over $\Lambda$ such that $\bar{F}_{11}=\sigma, \bar{F}_{22}=\tau, F_{33}=F_{22}$, and all other entries of $F$ are zero; as in the proof of "full" above, right multiplication by $F$ induces a $\Lambda$-homomophism $f: M \rightarrow M^{\prime}$ such that $\mathcal{E}(f)=(\sigma, \tau)$. But $(\sigma, \tau)$ is an isomorphism in the category $\mathcal{S}\left(1 \frac{1}{2}\right)$, and so $\sigma$ and $\tau$ are invertible matrices over the field $k$. Then the matrix $F$ is invertible, too. The same argument shows that the (block diagonal) matrix $F^{-1}$ induces a $\Lambda$-homomorphism $g: M \rightarrow M^{\prime}$ such that $\mathcal{E}(g)=\left(\sigma^{-1}, \tau^{-1}\right)=(\sigma, \tau)^{-1}$. Moreover, since $F F^{-1}$ is the identity map on $P$ (the projective cover of $M$ ), it follows that the induced map $f g$ is the identity map on $M$. Similarly, $g f$ is the identity map on $M^{\prime}$, and hence $f$ is an isomorphism between $M$ and $M^{\prime}$. 


\section{Klein and Dedekind-like rings.}

In this section, we show that a Klein ring is a homomorphic image of a Dedekind-like ring if and only if its residue field is imperfect (Theorem 5.2). Thus, there are Klein rings which are homomorphic images of Dedekind-like rings, and others which are not. For example, the group algebra $k G$, of the Klein 4-group $G$ over a field $k$ of characteristic 2, is a Klein ring (with residue field $k$ ); and $k G$ is a homomorphic image of a Dedekind-like ring if and only if $k$ is imperfect. Thus, as remarked in $\S 2$, we cannot simplify Theorem 2.10 by omitting Klein rings from its statement.

We conclude this section with an example of a Klein ring of characteristic 4 .

We begin by determining which Dedekind-like rings can be mapped onto Klein rings.

Theorem 5.1. Let $(\Lambda, \mathfrak{m}, k)$ be a Dedekind-like ring.

(i) If $\Lambda$ is split or a DVR, then $\Lambda$ cannot map onto a Klein ring.

(ii) If $\Lambda$ is unsplit with normalization $(\Gamma, \mathfrak{m}, F)$, then $\Lambda$ maps onto a Klein ring if and only if $F$ is an inseparable extension of $k$.

Proof. (i) Clearly a DVR, whose maximal ideal is principal, cannot map onto a Klein ring, whose maximal ideal requires two generators.

Thus let $(\Lambda, \mathfrak{m}, k)$ be a split Dedekind-like ring and $\Omega$ a Klein ring, and suppose, by way of contradiction, that there is a surjective ring homomorphism $\phi: \Lambda \rightarrow \Omega$. Then there would be a surjective homomorphism of the completion $\hat{\Lambda}$ onto $\Omega$, since artinian rings are already complete. Moreover, $\hat{\Lambda}$ is again split Dedekind-like, by Lemma 2.21. Therefore we may assume that $\Lambda$ is complete. As noted after (2.5.2), it follows that $\Lambda$ is strictly split.

Since $\Lambda$ is strictly split, its maximal ideal can be generated by two elements whose product is zero [Lemma 2.20]. Therefore the same is true of the maximal ideal $\mathfrak{n}$ of $\Omega$; say $\mathfrak{n}=(x, y)$. Since $x y=0$ and the square of every element of the maximal ideal of any Klein ring is zero, we have $\mathfrak{n}^{2}=0$. This is the desired contradiction, since the definition of "Klein ring" requires that $\mu_{\Omega}\left(\mathfrak{n}^{2}\right)=1$.

Before considering statement (ii) we prove two lemmas:

(5.1.1) Let $\Lambda$ be a Dedekind-like ring with normalization $\Gamma$, and $\varepsilon$ any element of $\Gamma-\Lambda$. Then $\Gamma=\Lambda+\Lambda \varepsilon$.

Since $\mathfrak{m}$ is an ideal of both rings $\Lambda$ and $\Gamma$, it suffices to prove this modulo $\mathfrak{m}$, whence it follows from the fact that $\Gamma / \mathfrak{m}$ is a 2 -dimensional vector space over the field $\Lambda / \mathfrak{m}=k$.

(5.1.2) Let $(\Lambda, \mathfrak{m}, k)$ be an unsplit Dedekind-like ring with normalization $(\Gamma, \mathfrak{m}, F)$, and $\varepsilon \in \Gamma-\Lambda$. If $F$ has characteristic 2 and is separable over $k$, we have $\Gamma=\Lambda+\varepsilon^{2} \Lambda$. 
To prove this it suffices, in view of (5.1.1), to prove that $\varepsilon^{2} \notin \Lambda$. Suppose, therefore that $\varepsilon^{2} \in \Lambda$. Then we have $F=k[\bar{\varepsilon}]$ where $\bar{\varepsilon}$ is the image of $\varepsilon$ in $F$, and $\bar{\varepsilon}^{2} \in k$. Since $F$ has characteristic 2 , we get the contradiction that $F$ is inseparable over $k$.

(ii) By Lemma 2.9, we can assume that $k$ and $F$ have characteristic 2 . Choose any $\varepsilon \in \Gamma-\Lambda$, recall that $\Gamma$ is DVR, and let $x$ be any $\Gamma$-generator of $\mathfrak{m}$ (i.e., $\mathfrak{m}=\Gamma x$ ).

First suppose that $F$ is separable over $k$. Multiplying the equation at the end of (5.1.2) by $x^{2}$ yields $\mathfrak{m}^{2}=\Lambda x^{2}+\Lambda(\varepsilon x)^{2}$. If there were a homomorphism of $\Lambda$ onto a Klein ring $(\Omega, \mathfrak{n}, k)$, the fact that the square of every element of $\mathfrak{n}$ is zero would then yield the contradiction $\mathfrak{n}^{2}=0$.

Suppose instead that $F$ is inseparable over $k$. We claim that $\Omega=\Lambda /\left(\mathfrak{m}^{3}+\right.$ $\left.\Lambda x^{2}\right)$ is a Klein ring.

Let $\mathfrak{n}$ be the maximal ideal of $\Omega$. Then we have $\mu_{\Omega}(\mathfrak{n})=2$ since $\mu_{\Lambda}(\mathfrak{m})=2$ [Lemma 2.7], and $\mathfrak{m}^{3}+\Lambda x^{2} \subseteq \mathfrak{m}^{2}=(\operatorname{rad} \Lambda) \mathfrak{m}$.

Next we show that $\mu_{\Omega}\left(\mathfrak{n}^{2}\right)=1$. Since $\Gamma$ is a DVR with residue field $F$, we have $\mathfrak{m}^{2} / \mathfrak{m}^{3} \cong F$ as $\Gamma$-modules, and hence as $k$-vector spaces, so that $\mathfrak{m}^{2} / \mathfrak{m}^{3}$ has dimension 2 as a vector space over $k$. Again, since $\Gamma$ is a DVR, we have $x^{2} \in \mathfrak{m}^{2}-\mathfrak{m}^{3}$, and therefore the dimension of the $k$-vector subspace $\left(\mathfrak{m}^{3}+\Lambda x^{2}\right) / \mathfrak{m}^{3}$ of $\mathfrak{m}^{2} / \mathfrak{m}^{3}$ equals 1 . But $\mathfrak{n}^{2}=\mathfrak{m}^{2} /\left(\mathfrak{m}^{3}+\Lambda x^{2}\right)$, so it also must have dimension 1 as a vector space over $k$, and therefore $\mu_{\Omega}\left(\mathfrak{n}^{2}\right)=1$, as desired.

Obviously $\mathfrak{n}^{3}=0$. We claim that every element of $\mathfrak{n}$ has square zero. First note that $\mathfrak{m}=\Gamma x=\Lambda x+\Lambda \varepsilon x$ and $\mathfrak{m}^{2}=\Gamma x^{2}=\Lambda x^{2}+\Lambda \varepsilon x^{2}$, by (5.1.1). Then take $a, b \in \Lambda$. Since $2 \in \mathfrak{m}$ (because $F$ has characteristic 2 ) and $\varepsilon^{2} \in \Lambda$ (because $F=k[\bar{\varepsilon}]$ is inseparable over $F$ ) we have

$$
(a x+b \varepsilon x)^{2}=a^{2} x^{2}+2(a b x)(\varepsilon x)+b^{2} \varepsilon^{2} x^{2} \in \Lambda x^{2}+\mathfrak{m}^{3}+\Lambda x^{2}
$$

which proves the claim.

Taking the opposite point of view, we determine which Klein rings are homomorphic images of Dedekind-like rings.

Theorem 5.2. If $(\Omega, \mathfrak{m}, k)$ is a Klein ring, then $\Omega$ is a homomorphic of a (necessarily unsplit) Dedekind-like ring if and only if its residue field $k$ is imperfect.

Proof. If the Klein ring $(\Omega, \mathfrak{m}, k)$ is a homomorphic image of the Dedekindlike ring $\Lambda$, then $k$ is the residue field of $\Lambda$. By Theorem 5.1, $\Lambda$ must be unsplit, and $k$ must be imperfect.

Conversely, suppose that $k$ is the imperfect residue field (necessarily of characteristic 2 , by Lemma 2.9$)$ of the Klein ring $(\Omega, \mathfrak{m}, k)$. We use the same idea as in the proofs of Propositions 3.4 and 3.6 to construct an unsplit Dedekind-like ring that maps onto $\Omega$. 
Fix generators $x$ and $y$ of $\mathfrak{m}$. By assumption $x^{2}=y^{2}=0$ and $\mathfrak{m}^{2}=\Omega x y \neq$ 0 . Using the Structure Theorem of Complete Local Rings [N, Theorem 31.1], there is a surjective homomorphism $\phi: V[[X, Y]] \rightarrow \Omega$ such that $\phi(X)=x$ and $\phi(Y)=y$, where $V[[X, Y]]$ is a formal power series ring, and either $V=k$, or $V$ is a complete DVR of characteristic 0 with residue field $k$ of characteristic 2 and the maximal ideal of $V$ is generated by 2 .

As in the proofs of Propositions 3.4 and 3.6, if $V$ is a field, we set $R=$ $V[[X, Y]]$, while if $V$ is not a field, we set $R=V[[X, Y]] /(2-(A X+B Y))$, where $A, B \in V[[X, Y]]$ are elements such that $\phi(2-(A X+B Y))=0$, changing notation so that $\phi$ is still the map from $R$ onto $\Omega$. Again, $\phi$ : $R \rightarrow \Omega$ is a surjective homomorphism such that $\phi(X)=x$ and $\phi(Y)=y$, where $R$ is a complete two-dimensional regular local ring with maximal ideal $\mathfrak{m}_{R}=(X, Y)$, and $\phi$ induces an isomorphism between the residue fields of $R$ and $\Omega$.

Because $k$ is imperfect of characteristic 2, not every element of $k$ is a square in $k$, so we can choose $C \in R$ whose coset modulo $\mathfrak{m}_{R}$ is not a square in $k$. We claim that $C X^{2}+Y^{2}$ is an irreducible element in the unique factorization domain $R$. Suppose, by way of contradiction, that $C X^{2}+Y^{2}$ is the product of two elements of $\mathfrak{m}_{R}$ :

$$
C X^{2}+Y^{2}=(S X+U Y)(T X+V Y)=S T X^{2}+(S V+U T) X Y+U V Y^{2}
$$

where $S, T, U, V \in R$. We know that $\mu_{R}\left(\mathfrak{m}_{R}^{2}\right)=3$ (by [AM, Theorem $11.22])$, and $\mathfrak{m}_{R}^{2}=\left(X^{2}, X Y, Y^{2}\right)$. Therefore the images of $X^{2}, X Y$, and $Y^{2}$ in $\mathfrak{m}_{R}^{2} / \mathfrak{m}_{R}^{3}$ are $k$-linearly independent. Comparing the coefficients of $Y^{2}$ on both sides of (5.2.1) therefore shows that $U V \equiv 1$ (all congruences in this part of the proof are modulo $\mathfrak{m}_{R}$ ). Therefore $U$ and $V$ are units in $R$. After multiplying the first and second factors in the middle part of (5.2.1) by $U^{-1}$ and $U$ respectively, and changing notation, we now have $U=1$ and $V \equiv 1$. Comparing coefficients of $X Y$ then shows that $S+T \equiv 0$, which is equivalent to $S \equiv-T$. But since $k$ has characteristic 2 , this is equivalent to $S \equiv T$. Comparing coefficients of $X^{2}$ therefore shows that $C \equiv S^{2}$, contrary to our choice of $C$ as an element of $R$ whose coset modulo $\mathfrak{m}_{R}$ is not a square. Thus, the claim is proved.

Set $\Lambda=R /\left(C X^{2}+Y^{2}\right)$, an integral domain because $C X^{2}+Y^{2}$ is an irreducible element of the unique factorization domain $R$. We claim that $\Lambda$ is unsplit Dedekind-like.

Let $c, x$, and $y(\in \Lambda)$ denote the cosets in $\Lambda$ of $C, X$, and $Y$, respectively, so that $c x^{2}+y^{2}=0$ in $\Lambda$. (Since we have no further need of $\Omega$, this duplication of earlier notation will cause no harm.) Let $\Gamma=\Lambda[y / x]$, a subring of the total quotient ring of $\Lambda$. Since $(y / x)^{2}=c \in \Lambda, \Gamma$ is an integral extension of $\Lambda$; in fact, $\mu_{\Lambda}(\Gamma) \leq 2$. 
We claim that $\mathfrak{m}_{\Lambda}$, which we write as $\mathfrak{m}$ from now on, is an ideal of $\Gamma$. Since $\mathfrak{m}=\Lambda x+\Lambda y$, it suffices to check that $x(y / x)$ and $y(y / x)$ are elements of $\mathfrak{m}$. The former is obvious, and the latter equals $\left(y^{2} / x^{2}\right) x=c x \in \mathfrak{m}$.

Let $F=\Gamma / \mathfrak{m}$, an algebra over its subfield $k=\Lambda / \mathfrak{m}$. We claim that $F$ is a 2-dimensional field extension of $k$. Let $\varepsilon=y / x$, and denote the natural images of $\varepsilon$ and $c$ in $F$ by $\bar{\varepsilon}$ and $\bar{c}$ respectively. Then $\bar{c} \in \Lambda / \mathfrak{m}=k$ and $\bar{\varepsilon}^{2}-\bar{c}=0$. By our choice of $C$, the element $\bar{c}$ is a nonsquare in $k$, and therefore the polynomial $Z^{2}-\bar{c}$ is irreducible over $k$. This shows that $F=k[\bar{\varepsilon}]$ is a 2-dimensional extension field of $k$ as claimed.

Next we claim that $\mathfrak{m}=\operatorname{rad}(\Gamma)$. Since $\mathfrak{m}$ is an ideal of $\Gamma$ the inclusion $(\subseteq)$ holds if we show that $1-m$ is invertible for every $m \in \mathfrak{m}$, and this holds since $\mathfrak{m}=\operatorname{rad}(\Lambda)$. The opposite inclusion holds because $F$ is a field.

Finally we claim that $\Gamma$ is a DVR (and is therefore the normalization of $\Lambda) . \quad \Gamma$ is a local ring because $\Gamma / \operatorname{rad}(\Gamma)$ is a field. Therefore it suffices to show that $\mathfrak{m}$ is a principal ideal of $\Gamma$. But $\mathfrak{m}=\Lambda x+\Lambda y$, so it suffices to observe that $y=(y / x) x \in \Gamma x$.

Remark 5.3 (Maximal tame?). Recall that, in the introduction, we called a ring "maximal tame" (following Ringel) if it is tame but not a proper homomorphic image of any other tame ring. In the commutative part of the situation considered by Ringel $[\mathbf{R}]$ - complete local rings that are algebras over their algebraically closed residue field - Klein rings turned out to be maximal tame. We compare this to the corresponding result in our more general situation.

Let $(\Omega, \mathfrak{m}, k)$ be a Klein ring, not necessarily an algebra over a field. Then $\Omega$ remains tame, by [KL2]. Moreover, $\Omega$ remains maximal tame if $k$ is perfect, because by our Theorem $5.2 \Omega$ is not a homomorphic image of a Dedekind-like ring.

On the other hand, if $k$ is imperfect of characteristic 2, then the result might change. In this case $\Omega$ is a homomorphic image of an unsplit Dedekind-like ring, say $(\Lambda, \mathfrak{m}, k)$, with normalization $(\Gamma, \mathfrak{m}, F)$ and $F$ inseparable over $k$. Here we are in the exceptional situation (1.0.1), in which we do not know whether $\Lambda$ is tame, wild, or neither. Thus, if $k$ is imperfect of characteristic 2 , we do not know whether $\Omega$ is maximal tame, although it is both tame and a homomorphic image of a Dedekind-like ring.

Example 5.4 (Klein ring of characteristic 4). Let $\Lambda_{p}=\mathbb{Z}[X] /\left(X^{2}, p^{2}\right)$, where $p$ is a prime number. In the case $p=2$, we claim that $\Lambda_{2}$ is a Klein ring of characteristic 4.

For every $p, \Lambda_{p}$ is a local artinian ring with maximal ideal $\mathfrak{m}_{p}=(x, \bar{p})$, where $x$ and $\bar{p}$ denote the images of $X$ and $p$ respectively, in $\Lambda_{p}$. Clearly, $\mathfrak{m}_{p}^{3}=0, \mu_{\Lambda}\left(\mathfrak{m}_{p}\right)=2$, and $\mu_{\Lambda}\left(\mathfrak{m}_{p}^{2}\right)=1$ (generated by $\bar{p} x$ ). 
We check that the square of every element of $\mathfrak{m}_{2}$ is zero. For any $w=$ $a x+\overline{2} b \in \mathfrak{m}_{2}$ we have $w^{2}=(a x+\overline{2} b)^{2}=a^{2} x^{2}+\overline{4} a b x+\overline{4} b^{2}=0$ in $\Lambda_{2}=$ $\mathbb{Z}[x] /\left(x^{2}, 4\right)$.

Note that $\Lambda_{p}$ cannot be a Klein ring when $p \neq 2$, because its characteristic is neither 2 nor 4 [Lemma 2.9]. In fact, $\Lambda_{p}$ is a homomorphic image of a Dedekind-like ring when $p \neq 2$. To prove this, note that $\Lambda_{p}$ does not map onto an artinian triad since $\mu\left(\mathfrak{m}_{p}\right)=2$, and does not map onto a Drozd ring since $\mu\left(\mathfrak{m}_{p}^{2}\right)=1$. Therefore, by our Ring-theoretic Dichotomy Theorem 3.1, $\Lambda_{p}$ is either a Klein ring (which we already know it is not) or a homomorphic image of a Dedekind ring (as claimed).

\section{Drozd rings and ramification.}

This section begins with two simple examples: A Drozd ring that is not an algebra over a field, and a ring that we call "superwild," something that cannot happen for algebras over fields. The main theorem of this section [Theorem 6.4] shows that every Drozd ring is a homomorphic image of a complete ramified order. Conversely, every complete ramified order is finitelength wild [Proposition 6.3]. We apply this to give the promised constructive definition of Drozd rings as a certain type of subring of local artinian principal ideal rings. Finally, we display in this form the two Drozd rings previously discussed.

Example 6.1. The simplest example of a Drozd ring that is not an algebra over a field, is the ring $A_{p}=\mathbb{Z}[X] /\left(X^{2}, p^{3}, p^{2} X\right)$, where $p$ denotes any prime number.

Example 6.2 (Superwild). The innocuous-looking (non-local) ring $\Lambda=$ $\mathbb{Z}[X] /\left(X^{2}\right)$ of Krull dimension 1 is superwild in the following sense: For every prime number $p, \Lambda$ is finite-length wild with respect to some residue field of characteristic $p$.

This holds because, for each prime number $p$, the ring $A_{p}$ in Example 6.1 is a Drozd ring with residue field $\mathbb{Z} /(p)$, and $A_{p}$ is a homomorphic image of $\Lambda$.

Three basic concepts in algebraic number theory, relating an order to an overorder, are those of splitting, residue-field growth, and ramification of a maximal ideal. The first two of these concepts occur in the present paper in the definitions of split and unsplit Dedekind-like rings, but ramification has yet to make an appearance. In fact, for orders ramification leads to wildness.

Proposition 6.3 (Ramification). Let $(\Lambda, \mathfrak{m}, k)$ be any complete local reduced ring that ramifies in its normalization $\Gamma$, in the sense that $\mathfrak{m}$ is contained in the square of some maximal ideal of $\Gamma$. Then $\Lambda$ is finite-length wild. 
Proof. Suppose that $\Lambda$ is not finite-length wild. First note that $\Lambda$ is not artinian, since then it would be a field. Therefore, by our Main Wildness Theorem 2.10, $\Lambda$ is a homomorphic image of a Dedekind-like ring, say $\Omega$. Moreover, $\Lambda \neq \Omega$ because the maximal ideal of a Dedekind-like ring never ramifies in its normalization. Thus the homomorphism $\Omega \rightarrow \Lambda$ is proper.

The Dedekind-like ring $\Omega$ cannot be unsplit, since then it is an integral domain; and all proper homomorphic images of noetherian domains of dimension 1 are artinian. Thus the only remaining possibility is that $\Omega$ is strictly split.

The normalization of $\Omega$ is therefore the direct sum of two DVRs, say $V_{1} \oplus V_{2}$. The imbedding $\Omega \subseteq V_{1} \oplus V_{2}$ is described in detail in Lemma 2.15. In particular, it is easy to see that $\Omega$ has only two dimension 1 prime ideals - the two projection maps $\Omega \rightarrow V_{i}$ - and therefore the only possible nonartinian, reduced homomorphic images of $\Omega$ are the coordinate rings $V_{i}$. But since $V_{i}$ is its own normalization, the maximal ideal of $V_{i}$ does not ramify in its normalization. This contradiction completes the proof.

It is an interesting fact that all Drozd rings arise in this way; that is, every Drozd ring is a homomorphic image of a ramified order.

Theorem 6.4 (Ramified onto Drozd). Let $(\Lambda, \mathfrak{n}, k)$ be a Drozd ring. Then $\Lambda$ is a homomorphic image of a completely ramified integral domain $\Omega$. In more detail, there is a commutative diagram of ring homomorphisms (6.4.1) in which:

(i) $(\Omega, \mathfrak{m}, k)$ is a complete local domain whose normalization is a DVR $(\Gamma,(z), k)$ such that $\mathfrak{m}=\Gamma z^{2}$

(ii) The vertical maps are surjections; and the left-hand vertical maps are the restrictions of $\rho^{\prime}$ and $\rho^{\prime \prime}$.

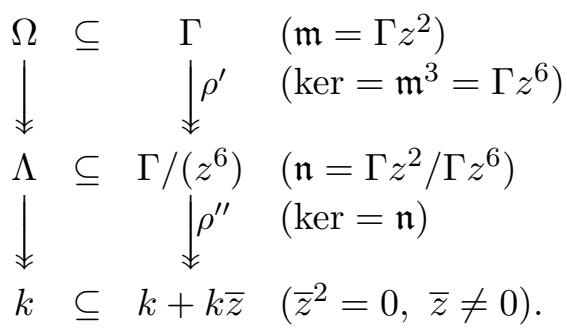

Proof. The approach is similar to that in the proofs of Propositions 3.4 and 3.6. We can write the maximal ideal of the Drozd $\operatorname{ring} \Lambda$ as $\mathfrak{n}=(x, y)$, where $x^{2}=y^{3}=0$ and $x y \neq 0$. As in the proofs of Propositions 3.4 and 3.6, by the Structure Theorem of Complete Local Rings [N, Theorem 31.1], there is a surjective homomorphism $\phi: V[[X, Y]] \rightarrow \Lambda$ such that $\phi(X)=x$ and $\phi(Y)=y$, where $V[[X, Y]]$ is a power series ring and either $V=k$ or $V$ is a complete DVR of characteristic 0 with residue field $k$. Moreover, in 
this latter case, $k$ has characteristic $p \neq 0$ and the maximal ideal of $V$ is generated by $p$.

If $V$ is a field, we set $R=V[[X, Y]]$, a complete, two-dimensional, regular local ring, with maximal ideal $(X, Y)$. If $V$ is a DVR, then again exactly as in the proofs of Propositions 3.4 and 3.6, we note that there are elements $A, B \in$ $V[[X, Y]]$ such that $\phi(p-(A X+B Y))=0$, and we set $R=V[[X, Y]] /(p-$ $(A X+B Y))$, a regular local ring by [AM, Theorem 11.22]. In this latter case, we change notation and let $\phi$ denote the induced map from $R$ onto $\Lambda$, and we let $X$ and $Y$ denote the cosets of $X$ and $Y$, respectively, in $R$. Thus, in either case we have a surjective homomorphism $\phi: R \rightarrow \Lambda$ such that $\phi(X)=x$ and $\phi(Y)=y$, where $R$ is a complete two-dimensional regular local ring and $(X, Y)$ is the maximal ideal of $R$.

Now $\phi\left(X^{2}-Y^{3}\right)=x^{2}-y^{3}=0$, and so $\phi$ induces a surjective homomorphism from $\Omega=R /\left(X^{2}-Y^{3}\right)$ onto $\Lambda$. We claim that $X^{2}-Y^{3}$ is irreducible in $R$, so that $\Omega$ is an integral domain.

Suppose, by way of contradiction, that $X^{2}-Y^{3}$ were reducible, say

$$
X^{2}-Y^{3}=(a X+b Y)(c X+d Y)=a c X^{2}+(a d+b c) X Y+b d Y^{2}
$$

for some elements $a X+b Y$ and $c X+d Y$ of the maximal ideal $(X, Y)$, with $a, b, c, d \in R$. Note that $(X, Y)^{2}=\left(X^{2}, X Y, Y^{2}\right)$ and, since $R$ is a regular local ring of dimension 2, the elements $X^{2}, X Y$, and $Y^{2}$ form a basis of $(X, Y)^{2} /(X, Y)^{3}$. Thus reading (6.4.2) modulo $(X, Y)^{3}$, we see that $a c$ is a unit in $R$, and hence $a$ and $c$ are units. Moreover, by this same reasoning, $1-a c \in(X, Y)$.

Similarly, looking at the coefficient of $Y^{2}$ and reading (6.4.2) modulo $(X, Y)^{3}$ shows that $b d \in(X, Y)$, and hence at least one of $b$ and $d$ is in $(X, Y)$. But then both $b$ and $d$ must be in $(X, Y)$, because otherwise, (using the fact that $a$ and $c$ are both units) the coefficient of $X Y$ would be a unit on the right-hand side of (6.4.2) but zero on the left-hand side.

We have $(X, Y)^{3}=\left(X^{3}, X^{2} Y, X Y^{2}, Y^{3}\right)$ and the elements $X^{3}, X^{2} Y$, $X Y^{2}$, and $Y^{3}$ form a basis of $(X, Y)^{3} /(X, Y)^{4}$ (again because $R$ is a regular local ring of dimension 2). Rearranging (6.4.2), we get that

$$
(1-a c) X^{2}-Y^{3}-(a d+b c) X Y \equiv 0
$$

$\left(\bmod (X, Y)^{4}\right)$

since $b$ and $d$ are in $(X, Y)$, so that $b d Y^{2} \in(X, Y)^{4}$. Since $1-a c, b$, and $d$ are all in $(X, Y)$ we can substitute expressions of the form $1-a c=e X+f Y$ and $a d+b c=g X+h Y$ into (6.4.3). Since the coefficient of $Y^{3}$ is a unit, this gives a nontrivial linear relation among the basis elements of $(X, Y)^{3} /(X, Y)^{4}$, and this contradiction proves our claim that $X^{2}-Y^{3}$ is irreducible in $R$. Therefore $\Omega$ is an integral domain.

Let $s$ and $t$ denote the cosets of $X$ and $Y$, respectively, in $\Omega=R /\left(X^{2}-\right.$ $\left.Y^{3}\right)$, and recall that $\mathfrak{m}$ denotes the maximal ideal of $\Omega$, so that $\mathfrak{m}=(s, t)$. Since $s^{2}=t^{3}$, we have $(s / t)^{2}=t$ in the quotient field of $\Omega$. It follows that 
$z=s / t$ is integral over $\Omega$, and hence $\Gamma=\Omega[z]$ is an integral extension of $\Omega$. Moreover, since $z \cdot t=s \in \mathfrak{m}$ and $z \cdot s=s^{2} / t=t^{3} / t=t^{2} \in \mathfrak{m}$, we have that $\mathfrak{m}$ is an ideal of $\Gamma$ also. Since $\mathfrak{m}$ is the maximal ideal of $\Omega$, it follows that $\mathfrak{m}$ is the conductor from $\Gamma$ to $\Omega$. Also, $\mathfrak{m}=\operatorname{rad}(\Omega)$, and $\Gamma$ is finitely generated as an $\Omega$-module, so $\mathfrak{m} \subseteq \operatorname{rad}(\Gamma)$. We claim that

$$
\mu_{\Omega}(\mathfrak{m})=2, \quad z^{2} \in \mathfrak{m}, \quad z \notin \mathfrak{m} .
$$

We already have that $\mathfrak{m}=(s, t)$ as an ideal of $\Omega$. The fact that two generators are required follows from the fact that $\mathfrak{m}$ maps onto the maximal ideal of the Drozd ring $\Lambda$. Next, note that $z^{2}=(s / t)^{2}=t \in \mathfrak{m}$. Finally, if $z$ were an element of $\mathfrak{m}$, then $s / t=z$ would imply that $s=t z \in \mathfrak{m}^{2}$, which would make the ideal $\mathfrak{m}=(s, t)$ of $\Omega$ principal, contrary to what was just shown.

Since $\Gamma=\Omega[z],(6.4 .4)$ yields

$$
\Gamma / \mathfrak{m}=k+k \bar{z} \quad \text { with } \quad \bar{z}^{2}=0 \text { and } \bar{z} \neq 0 .
$$

This yields the first equality in

$$
\operatorname{rad}(\Gamma)=\mathfrak{m}+\Gamma z=\Gamma z .
$$

The second equality holds since $s=z \cdot t \in \Gamma z$ and $t=z^{2} \in \Gamma z$.

The fact that $\operatorname{rad}(\Gamma / \mathfrak{m})=(\bar{z})$ is the maximal ideal of $\Gamma / \mathfrak{m}$ now shows that $\operatorname{rad}(\Gamma)=\Gamma z$ is the maximal ideal of $\Gamma$; that is, $\Gamma$ is a local domain with principal maximal ideal $\Gamma z$. Therefore $\Gamma$ is a DVR and hence is the normalization of $\Omega$.

Next we note that, since $\Gamma$ is a DVR with maximal ideal $\Gamma z,(6.4 .4)$ implies that $\mathfrak{m}=\Gamma z^{2}$.

We have now completed the proof of the top line in diagram (6.4.1). We also have established the existence of a surjective ring homomorphism $\rho^{\prime}: \Omega \rightarrow \Lambda$. The map $\rho^{\prime}$ takes $\mathfrak{m}=\mathfrak{m}_{\Omega}$ onto $\mathfrak{n}=\mathfrak{m}_{\Lambda} ;$ and $\mathfrak{n}^{3}=0$ since $\Lambda$ is a Drozd ring. We conclude that $\mathfrak{m}^{3} \subseteq \operatorname{ker} \rho^{\prime}$; we claim that equality holds.

It suffices to prove that the composition length of $\Omega / \mathrm{m}^{3}$ equals the length of $\Lambda$, namely 5 . The length of $\Omega / \mathrm{m}^{3}$ is at least 5 , since $\Omega$ maps onto $\Lambda$. Therefore it suffices to prove that the length of the $\Omega$-module $\Omega / \mathfrak{m}^{3}$ is less than 6 .

Since $\Omega$ is properly contained in $\Gamma$, it suffices to show that the length of the $\Omega$-module $\Gamma / \mathfrak{m}^{3}$ equals 6 . Now, the simple $\Gamma$-module $k$ remains simple as an $\Omega$-module, by (6.4.5) and the fact that $\Omega / \mathfrak{m}=k$. Therefore, it suffices to show that the length of the $\Gamma$-module $\Gamma / \mathfrak{m}^{3}$ equals 6 . This follows from the fact that $\Gamma$ is a DVR whose maximal ideal $\Gamma z$ satisfies $\Gamma z^{2}=\mathfrak{m}$.

We have now established the existence of the top commutative square in (6.4.1). The existence of the bottom commutative square now follows immediately from (6.4.5), completing the proof of the theorem.

By an AVR (artinian valuation ring) of length $n$ we mean a local principal ideal ring $(V,(z), k)$, with lattice of ideals $V \supset V z \supset V z^{2} \supset \ldots \supset V z^{n}=0$ 
for some $n$. We say that $V$ contains its residue field if $V$ contains a subfield that maps isomorphically onto $k$ via the natural homomorphism $V \rightarrow k$.

We are now ready to give our constructive definition of "Drozd ring" as a certain type of subring of an AVR of length 6. This AVR seems to be a kind of "artinian normalization" of the Drozd ring. In more detail, the Drozd ring $\Lambda$ is the pullback of square (6.5.1), and if we replace the AVR by a DVR that contains its residue field (and delete the condition " $x^{2}=0$ "), we get the ordinary conductor square for a simple cusp whose normalization is the DVR.

Theorem 6.5 (Constructive Definition of Drozd Rings). Let $(V,(z), k)$ be an AVR of length 6 such that the ring $V /\left(z^{2}\right)$ contains its residue field (and hence $V /\left(z^{2}\right)=k+k \bar{z}$ with $\bar{z}^{2}=0$ and $\left.\bar{z} \neq 0\right)$, and let $\Lambda$ be the pullback of diagram (6.5.1). Then:

(i) $\Lambda$ is a Drozd ring, and every Drozd ring arises in this way.

(ii) $V=\Lambda+\Lambda z$ and $\mathfrak{m}_{\Lambda}=\Lambda x+\Lambda y$, where $x=z^{3}, y=z^{2}, x \in \mathfrak{m}_{V}-\mathfrak{m}_{V}^{2}$, and $x^{2}=0$.

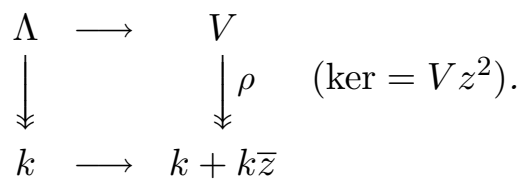

Proof. The fact that every Drozd ring arises in this way is expressed in the bottom square of diagram (6.4.1).

Conversely, given that $\Lambda$ is the pullback of diagram (6.5.1), we want to prove that $\Lambda$ is a Drozd ring satisfying conditions (ii).

Since the image of the left-hand vertical map in (6.5.1) is the field $k$ and the kernel of this map is nilpotent, we see that $\Lambda$ is a local ring with maximal ideal $\mathfrak{m}_{\Lambda}=V z^{2}$ and residue field $k$. Moreover, $V=\Lambda+\Lambda z$ holds because it obviously holds modulo $V z^{2}$. Multiplying by $z^{2}$ then shows

$$
\mathfrak{m}_{\Lambda}=\Lambda z^{2}+\Lambda z^{3} \text {. }
$$

To see that $\mathfrak{m}_{\Lambda}$ requires two generators (as a $\Lambda$-module), note that $\mathfrak{m}_{\Lambda} / \mathfrak{m}_{\Lambda}^{2}=$ $\left(V z^{2}\right) /\left(V z^{4}\right)$. Since $V$ is an AVR, this is $V$-isomorphic to $V /\left(V z^{2}\right)$, a $k$ vector space of dimension 2 by (6.5.1). Similarly, to see that $\mathfrak{m}_{\Lambda}^{2}$ requires two generators as a $\Lambda$-module, we note that $\mathfrak{m}_{\Lambda}^{2} / \mathfrak{m}_{\Lambda}^{3}=V z^{4}$ has $k$-dimension 2 .

Thus, to complete the proof that $\Lambda$ is a Drozd ring, it suffices to note that $x=z^{3}$ is an element of $\mathfrak{m}-\mathfrak{m}^{2}=V z^{2}-V z^{4}$ (obvious, since $V$ is an AVR), $x^{2}=0$, and $\mathfrak{m}_{\Lambda}^{3}=V z^{6}=0$.

Examples 6.6. We display, as subrings of their AVR "normalization" $V$, the two Drozd rings explicitly discussed in this paper: 
(i) $\Lambda=k+k x+k y+k x y+k y^{2}$, the Drozd $k$-algebra originally studied by Drozd. Here $k$ is any field, and all monomials in $x$ and $y$ other than the displayed ones equal 0 . Choose $V=k[z]$ where $z$ satisfies the defining relation $z^{6}=0$. Then $\Lambda$ is the $k$-subalgebra with basis $1, z^{2}=y, z^{3}=$ $x, z^{4}=y^{2}, z^{5}=x y$.

(ii) $\Lambda=\mathbb{Z}[X] /\left(X^{2}, p^{3}, p^{2} X\right)$, the Drozd ring in Example 6.1.

Choose $V=\overline{\mathbb{Z}}_{p^{3}}[W] /\left(W^{6}, W^{2}-\bar{p}\right)$ where $\overline{\mathbb{Z}}_{p^{3}}$ denotes the integers modulo $p^{3}$ and $W$ is an indeterminate. Letting $w$ be the natural image of $W$ in $V$, we see that $V$ is an AVR of length 6 with composition series

$$
V \supset V w \supset V w^{2}=V \bar{p} \supset V w^{3}=V p w \supset V w^{4}=V \bar{p}^{2} \supset V w^{5}=V \bar{p}^{2} w \supset 0
$$

and $V / V w^{2}$ contains its residue field $\overline{\mathbb{Z}}_{p}$.

The subring of $V$ additively spanned by the monomials $1, w^{2}=\bar{p}, w^{3}=$ $\bar{p} w, w^{4}=\bar{p}^{2}, w^{5}=\bar{p}^{2} w$ is isomorphic to $\Lambda$, under the correspondence $\bar{p} \rightarrow \bar{p}$ and $\bar{p} w \rightarrow x$.

\section{Infinite lattice type.}

If a local ring $\Lambda$ is reduced (no nonzero nilpotent elements), then submodules of free $\Lambda$-modules of finite rank are often called lattices. (This is equivalent to the definition of "lattice" used in integral representation theory.) $\Lambda$ is said to have infinite lattice type, or less precisely, "infinite representation type," if it has infinitely many indecomposable lattices. There exist many such $\Lambda$ with infinite but tame lattice type. However, in the present context, such commutative noetherian rings are finite-length wild.

Theorem 7.1. Let $(\Lambda, \mathfrak{m}, k)$ be a complete local reduced ring. If $\Lambda$ has infinite lattice type, then $\Lambda$ is finite-length wild.

Proof. First recall that a reduced, non-artinian, proper homomorphic image of a strictly split or unsplit Dedekind-like ring must be a DVR.

Next note that $\Lambda$ is not artinian, since then it would be a direct product of fields, and hence have finite lattice type.

It is easiest to prove the contrapositive of our theorem, so suppose that $\Lambda$ is not finite-length wild. Then by our Main Wildness Theorem 2.10 and the fact that $\Lambda$ is not artinian, $\Lambda$ must be a homomorphic image of a complete split (and hence strictly split) or unsplit Dedekind-like ring $\Omega$. Since $\Lambda$ is reduced, it must therefore be either one of these types of Dedekind-like rings or else (see the first paragraph of this proof) a DVR.

Since DVRs are well-known to have finite lattice type, we may suppose that $\Lambda$ is split or unsplit Dedekind-like, with normalization $\Gamma$. Then $\mu_{\Lambda}(\Gamma / \Lambda)=1$ and $\mu_{\Lambda}(\operatorname{rad}(\Gamma / \Lambda))=0$ (since $\mathfrak{m} \cdot \Gamma / \Lambda=0$, so that $\Gamma / \Lambda$ is a $k$-module, hence a simple $\Lambda$-module). Therefore $\Lambda$ satisfies the DrozdRoiter conditions and hence, by a result of Cimen and Wiegand (extending 
earlier results of Drozd, Roiter, Jacobinski, Green, and Reiner on lattices over orders) must have finite lattice type $[\mathbf{C W W}]$.

\section{References}

[AM] M.F. Atiyah and I.G. MacDonald, Introduction to Commutative Algebra, Addison-Wesley, Reading, 1969, MR 39 \#4129, Zbl 175.03601.

[B] S. Brenner, Decomposition properties of some small diagrams of modules, Symposia Mathematica, 13 (1974), 127-141, MR 50 \#13159, Zbl 289.16019.

[CWW] N. Cimen, R. Wiegand and S. Wiegand, One-dimensional rings of finite representation type, Abelian groups and modules (Padova, 1994), 95-121, Math. Appl., 343, Kluwer Acad. Publ., Dordrecht, 1995, MR 97a:13014, Zbl 856.13006.

[CB] W.W. Crawley-Boevey, On tame algebras and BOCS's, Proc. LMS, 56 (1988), 451-483, MR 89c:16028, Zbl 661.16026.

[CR1] C.W. Curtis and I. Reiner, Methods of Representation Theory, with Applications to Finite Groups and Orders, I, John Wiley and Sons, New York, 1981, MR 82i:20001, Zbl 469.20001.

[D] Yu.A. Drozd, Representations of commutative algebras (Russian), Funkstional'nyi Analiz i Ego Prilozheniya, 6 (1972), 41-43; English Translation in 'Functional Analysis and its Applications', 6 (1972), 286-288, MR 7 \#280, Zbl 289.13009.

[D2] - Finite modules over pure noetherian algebras, Proc. Steklov Institute of Math., 4 (1991), 97-108.

[GLW] R.M. Guralnick, L.S. Levy and R.B. Warfield, Jr., Cancellation counterexamples in Krull dimension 1, Proceedings Amer. Math. Soc., 109 (1990), 323-326, MR 90i:13007, Zbl 706.13010.

[Hu] T.W. Hungerford, On the structure of principal ideal rings, Pacific J. Math., 25 (1968), 543-547, MR 37 \#2744, Zbl 157.08503.

[K] L. Klingler, Modules over the integral group ring of a nonabelian group of order pq, Mem. Amer. Math. Soc., 59 (1986), MR 87g:16015, Zbl 598.16014.

[KL2] L.C. Klingler and L.S. Levy, Representation type of commutative noetherian rings II: Local tameness, Pacific J. Math., 200 (2001), 387-483.

[KL3] _ Representation type of commutative noetherian rings III: global wildness, in preparation.

[KL4] _ Representation type of commutative noetherian rings IV: global tameness, in preparation.

[L1] L.S. Levy, $Z G_{n}$-modules, $G_{n}$ cyclic of square-free order n, J. Alg., 93 (1985), 354-375, MR 87k:20017b, Zbl 596.13010.

[L2] _ Modules over Dedekind-like rings, J. Alg., 93 (1985), 1-116, MR 87k:20017a, Zbl 564.13010.

[N] M. Nagata, Local Rings, John Wiley and Sons, New York, 1962, MR 27 \#5790, Zbl 123.03402.

[Nz] L.A. Nazarova, Representations of quivers of infinite type, Izv. Akad. Nauk SSSR, Ser Mat., 37(4) (1973); English transl: Math. USSR Izvestija, 7(4) (1973), 749792, MR 49 \#2785, Zbl 343.15004. 
[NR] L.A. Nazarova and A.V. Roiter, Finitely generated modules over a dyad of two local rings and finite groups with an abelian normal divisor of Index $p$, Zap. Nauch. Sem. Leningrad Odtel. Mat. Inst. Steklov (LOM1), Izv. Akad. Nauk SSSR, Ser Mat. 33(1) (1969); English transl: Math. USSR Izvestija, 3(1) (1969), 65-89, MR 41 \#5479, Zbl 207.04803.

[NRSB] L.A. Nazarova, A.V. Roiter, V.V. Sergeichuk and V.M. Bondarenko, Application of modules over a dyad for the classification of finite p-groups that have an abelian subgroup of index $p$ and of pairs of mutually annihilating operators (Russian), Zap. Nauch. Sem. Leningrad Odtel. Mat. Inst. Steklov (LOM1), 28 (1972), 69-92; English transl: J. Soviet Math., 3 (1975), 636-653, MR 48 \#11288, Zbl 346.13005.

[R] K.M. Ringel, The representation type of local algebras, Springer Lecture Notes in Mathematics, 488 (1975), 282-305, MR 52 \#3241, Zbl 348.16013.

[S] E. Steinitz, Rechteckige Systeme und Moduln in Algebraicher Zahlkörper I, II, Math. Ann., 71 (1911), 328-354; 72 (1912), 297-345.

Received September 9, 1999 and revised November 29, 2000. Levy's research was partially supported by an NSA grant.

Mathematics Department

Florida Atlantic University

Boca Raton, Florida 33431-0991

E-mail address: klingler@fau.edu

Mathematics Department

UNIVERSITY OF WISCONSIN

MAdison, Wisconsin 53706-1388

E-mail address: levy@math.wisc.edu 